\title{
REINFORCEMENT LEARNING For Decentralized Stochastic Control
}

\author{
by
}

\section{Bora YongACOGLU}

\author{
A thesis submitted to the \\ Department of Mathematics and Statistics \\ in conformity with the requirements for \\ the degree of Master of Science \\ Queen's University \\ Kingston, Ontario, Canada
}

August 2018

Copyright (c) Bora Yongacoglu, 2018 


\begin{abstract}
We consider decentralized optimization of a controlled stochastic system where a finite number of decision makers seek to arrive at optimal policies using only local measurements and cost realizations. For such a context, a recent result in the literature established convergence to person-by-person-optimal policies (and a class of Nash equilibrium policies in the game theoretic setup). Our main theorem is a convergence result to global optimality in a team-theoretic setup, and not only person-by-person optimality, with arbitrarily high probability.
\end{abstract}




\section{Acknowledgments}

I am most grateful for my family and my relatives. Throughout all my pursuits, they have given me their unwavering support.

I wish to thank my advsior, Professor Serdar Yüksel, for his guidance and patience.

I would also like to thank Professors Gürdal Arslan, Tamás Linder, and Thomas Barthelmé for their time and attention while taking part in my thesis committee. 


\section{Contents}

Abstract

$\begin{array}{ll}\text { Acknowledgments } & \text { ii }\end{array}$

Contents

Chapter 1: Introduction 1

1.1 Motivation . . . . . . . . . . . . . . . . . 1

1.2 Organization of Thesis . . . . . . . . . . . . . . . 3

Chapter 2: Background 4

2.1 Markov Decision Processes . . . . . . . . . . . . . . . 5

2.2 Reinforcement Learning for MDPs . . . . . . . . . . . . . . . . . . . . 11

2.3 Stochastic Games . . . . . . . . . . . . . . . . . . . . . 15

2.4 Literature Review . . . . . . . . . . . . . . . . . . . . . 19

Chapter 3: $\quad$ Main Results 22

3.1 The Algorithms in Pseudocode . . . . . . . . . . . . . . 23

3.2 Theorem Statement . . . . . . . . . . . . . . . . . . 30

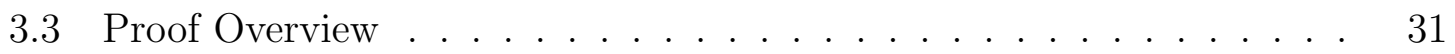

3.4 A Markov Chain to be Used for Comparison . . . . . . . . . . . . . 35

3.5 Results on Similar Matrices . . . . . . . . . . . . . . . . . . 39

3.6 Parameter Restriction and Supporting Results . . . . . . . . . . . . 45

3.7 Theorem and Proof . . . . . . . . . . . . . . . . 62

$\begin{array}{lll}\text { Chapter 4: } & \text { Simulation Study } & 65\end{array}$

$\begin{array}{lll}\text { Chapter 5: } & \text { Summary and Conclusions } & 74\end{array}$

$\begin{array}{ll}\text { Bibliography } & 75\end{array}$ 


\section{Chapter 1}

\section{Introduction}

\subsection{Motivation}

A cooperative system is a strategic environment in which (i) outcomes depend jointly on the actions of multiple decision making agents, and (ii) all of the agents have aligned preferences. That is, any one agent's preferences over the possible outcomes is the same as any other agent's preferences over the possible outcomes. The umbrella of cooperative systems covers any setting in which multiple agents are given the same task, find themselves on the same team in some sense, or incur identical costs.

Cooperative systems are ubiquitous in engineering and the social sciences: in eco-

nomics, they arise as coordination games. For example, see [4] and the references therein. In control, they take the form of distributed or decentralized problems, where there are multiple controllers. See [18] for a full treatment. In computer science, cooperative systems arise in machine learning among others areas.

Coordinating the behaviour of the many agents to achieve stable or optimal system 
performance is a common goal when studying cooperative systems. In instances where centralization of the system is possible, a central planner may achieve coordination by treating the problem as a regular (single agent) control or optimization problem, and then issuing commands to the agents based on the analysis of the system.

However, problems in cooperative systems become still more difficult when there are obstacles to centralization. Among others, two notable obstacles worth considering are information constraints and communication constraints. Information constraints can take a number of forms, including uncertainty about the environment, about other players, about observations/feedback, or about one's own preferences over outcomes. Communication constraint can take the form of noisy or non-existent channels, among other forms.

In many interesting applications, centralization is infeasible. When too much information needs to be communicated, stored, and processed, the job of the central planner may become computationally intractable. In other instances-for example, when information constraints are such that agents do not know they are in a cooperative setting-agents might be unwilling or untrusting of a central planner, and will not communicate the necessary information.

In such settings, there is a need for decentralized processes that reliably lead to good system performance. This brings us to the aim of the work: we seek to develop learning algorithms that (i) reliably coordinate the behaviour of agents to the optimal behaviour, and (ii) have desirable features: the learning algorithms we wish to devise are individually rational (in particular, their justification should not rely on an agent's knowing that they are in a cooperative system), that share information minimally 
across agents, and that require minimal understanding of the game a priori.

The contribution of this thesis is to provide such a learning algorithm, and to prove rigorously that it performs well in a very general set of decentralized control problems. While there are existing learning algorithms that achieve coordination, to the best of our knowledge they either depend on considerably greater information, or otherwise do not have any guarantees for optimality in the general setting we consider. In our setting with restricted communication and limited local information, Algorithm B presented here may be the first rigorous result for optimal coordination in stochastic team games.

\subsection{Organization of Thesis}

In chapter 2 , we provide the necessary background and survey the literature relevant to our problem.

In chapter 3, we state and prove our main theorem. The principal contributions of the thesis are here. This chapter also contains multiple supporting results, and the proof technique of approximating a non-Markovian process by a Markovian process might be of value independent of the main result.

In chapter 4 , we provide a toy example, and show results of a simulation study. The numerical results support the theoretical result in chapter 3. 


\section{Chapter 2}

\section{Background}

\section{Chapter Outline}

This chapter covers the necessary theory and definitions, and then reviews some relevant literature.

In section 2.1, we begin by introducing a one agent model (Markov Decision Processes) and providing useful facts in that setting.

In section 2.2, we discuss Q-learning, a well-studied reinforcement learning method used for solving Markov decision processes, and we state and prove some useful propositions.

In section 2.3, we introduce stochastic games and define the relevant objects. A stochastic game is a multi-agent generalization of Markov decision processes, and is the model used throughout this thesis.

After the objects of study have been defined in the previous sections, section 2.4 surveys the literature. 


\subsection{Markov Decision Processes}

Let us first consider a setting in which there is only one decision making agent, rather than many. In this setting, communication issues need not be addressed, but informational constraints may still exist: the agent may not understand the environment, the consequence of their actions, or the payoff structure.

Markov decision processes (MDPs) constitute one popular and well-studied model for single-agent decision making. In an MDP, an agent takes actions that probabilistically influence both the received payoffs and the evolution of the state variable. MDPs are often used to model situations in which there is a trade-off between short- and longterm cost.

Definition: A finite, discounted Markov Decision Process is a decision problem with the following characteristics:

- a single decision making agent (DM)

- a finite set of states $\mathbb{X}$

- a finite set of control actions $\mathbb{U}$.

- a one-stage cost function $c: \mathbb{X} \times \mathbb{U} \rightarrow \mathbb{R}$

- a discount factor $\beta \in(0,1)$

- a probabilistic transition kernel $P[\cdot \mid x, u]$ for determining the state for the next time stage

When facing such an MDP, the DM must choose a policy, i.e. a rule for how to select actions given the information available, with the aim of minimizing the expected 
long-term discounted cost.

We define the long term cost associated to a policy $\pi$ in a state $x$ as follows:

$$
J_{x}(\pi)=E\left[\sum_{k \geq 0} \beta^{k} c\left(x_{k}, u_{k}\right) \mid x_{0}=x\right]
$$

where $u_{k}$ is the action generated by the policy $\pi$ in the $k^{t h}$ period.

\section{Admissible, Markov, and Stationary Policies}

Let $\mathcal{F}_{t}$ denote the $\sigma$-algebra generated by all of the information available to the DM at time $t$.

Definition: We say that a policy $\pi=\left(\pi_{t}\right)_{t \in \mathbb{N}}$ is admissible if for every index $t \in \mathbb{N}$, we have (i) $\pi_{t}$ is $\mathcal{F}_{t}$-measurable, and (ii) the image of $\pi_{t}$ is contained in $\mathcal{P}(\mathbb{U})$, the set of probability measures on $\mathbb{U}$. We denote the set of all admissible policies by $\Delta$.

Definition: We say that a policy $\pi=\left(\pi_{t}\right)_{t \in \mathbb{N}}$ is deterministic Markov if $\pi_{t}: \mathbb{X} \rightarrow \mathbb{U}$ for every index $t \in \mathbb{N}$. We will denote the set of all Markov policies by $M$.

Definition: We say that a deterministic Markov policy $\pi=\left(\pi_{t}\right)_{t \in \mathbb{N}}$ is stationary if $\pi_{t} \equiv \pi: \mathbb{X} \rightarrow \mathbb{U}$. That is, if the action selected is only a function of the current state, and the action selected is independent of selection time. We denote the set of all deterministic stationary policies by $\Pi$.

For these definitions and more, see p. 456-457 in [18] or p. 12 in [17]. 


\section{Two Results on Optimal Policies}

With the relevant definitions and notation established, we can discuss some of the well-known facts about MDPs. Notably, there exists an optimal policy in the set of deterministic stationary policies:

Proposition 2.1:

$$
\exists \pi^{*} \in \Pi \text { such that } J_{x}\left(\pi^{*}\right)=\inf _{\gamma \in \Delta} J_{x}(\gamma) \text { for every state } x \in \mathbb{X}
$$

The reader is referred to 5.5.1 in [17] for a proof, and to [6] for a lengthier treatment.

Next, we recall the dynamic programming operator $T: \mathbb{R}^{\mathbb{X}} \rightarrow \mathbb{R}^{\mathbb{X}}$. Let $V \in \mathbb{R}^{\mathbb{X}}$

$$
\begin{aligned}
T V(x) & =\min _{u \in U}\left[E(c(x, u))+\beta \sum_{y \in \mathbb{X}} P(y \mid x, u) V(y)\right] \\
& =\min _{u \in \mathbb{U}} E[c(x, u)+\beta V(s(x))]
\end{aligned}
$$

and where $s(x)$ denotes the random state that follows $x$ after playing $u$.

Lemma 2.2: For any $V, W \in \mathbb{R}^{\mathbb{X}}$, we have $\|T W-T V\|_{\infty} \leq \beta\|W-V\|_{\infty}$.

Proof of lemma 2.2: We will show that $|T W(x)-T V(x)| \leq \beta|W(x)-V(x)|$ for every 
state $x$

$$
\begin{aligned}
& |T W(x)-T V(x)| \\
& =\left|\min _{u \in \mathbb{U}}\left[E(c(x, u))+\beta \sum_{y \in \mathbb{X}} P(y \mid x, u) W(y)\right]-\min _{z \in \mathbb{U}}\left[E(c(x, z))+\beta \sum_{y \in \mathbb{X}} P(y \mid x, u) V(z)\right]\right| \\
& \leq \max _{u \in \mathbb{U}}\left|\left[E(c(x, u))+\beta \sum_{y \in \mathbb{X}} P(y \mid x, u) W(y)\right]-\left[E(c(x, u))+\beta \sum_{y \in \mathbb{X}} P(y \mid x, u) V(y)\right]\right| \\
& =\max _{u \in \mathbb{U}}\left|\beta \sum_{y \in \mathbb{X}} P(y \mid x, u)(W(y)-V(y))\right| \\
& \leq \beta\|W-V\|_{\infty}
\end{aligned}
$$

Thus, $T$ is a contraction. Since $|\mathbb{X}|$ is finite, Banach's fixed point theorem allows us to conclude that $T$ is continuous, and has a unique fixed point, denoted $V^{*}$.

One can recover an optimal policy from $V^{*}$, using this very useful characterization of optimal stationary policies.

Proposition 2.3: Let $\Pi^{*}=\left\{\pi \in \Pi: J_{x}(\pi)=\inf _{\gamma \in \Delta} J_{x}(\gamma) \quad \forall x \in \mathbb{X}\right\}$ denote the set of optimal deterministic policies, and let $\pi \in \Pi$. Then, $\pi \in \Pi^{*}$ if and only if

$$
E[c(x, \pi(x))]+\beta \sum_{y \in \mathbb{X}} P(y \mid x, y) V^{*}(y)=\min _{u \in \mathbb{U}} E[c(x, u)]+\beta \sum_{y \in \mathbb{X}} P(y \mid x, u) V^{*}(y)
$$

holds for every state $x$.

The proof of this proposition follows immediately from the following three lemmas (Lemmas 2.4-2.6).

Lemma 2.4: For any stationary policy $\gamma \in \Pi$, we have $J_{x}(\gamma) \geq V^{*}(x)$ for every state $x$. 
Proof of lemma 2.4: Consider the vector $W \in \mathbb{R}^{\mathbb{X}}$ given by $W(x)=J_{x}(\gamma)$. We will show that $T^{k} W(x) \leq J_{x}(\gamma)$ for any $k \in \mathbb{N}$, and then conclude $V^{*}(x) \leq J_{x}(\gamma)$.

Let $s(x)$ denote the successor state to $\mathrm{x}$. The case $k=1$ holds for any $x \in \mathbb{X}$ :

$$
\begin{aligned}
T W(x) & =\min _{u \in \mathbb{U}} E\left[c(x, u)+\beta W(s(x)) \mid x_{0}=x, u_{0}=u\right] \\
& =\min _{u \in \mathbb{U}} E\left[c(x, u)+\beta J_{s(x)}(\gamma) \mid x_{0}=x, u_{0}=u\right] \\
& \leq E\left[c(x, \gamma(x))+\beta J_{s(x)}(\gamma) \mid x_{0}=x, u_{0}=\gamma(x)\right] \\
& =J_{x}(\gamma)
\end{aligned}
$$

For the induction step, assume that $T^{n} W(x) \leq J_{x}(\gamma) \forall x \in \mathbb{X}$. Then,

$$
\begin{aligned}
T^{n+1} W(x) & =T\left(T^{n} W\right)(x) \\
& =\min _{u \in \mathbb{U}} E\left[c(x, u)+\beta T^{n} W(s(x)) \mid x_{0}=x, u_{0}=u\right] \\
& \leq \min _{u \in \mathbb{U}} E\left[c(x, u)+\beta J_{s(x)} \mid x_{0}=x, u_{0}=u\right] \quad \text { (by the induction hypothesis) } \\
& \leq E\left[c(x, \gamma(x))+\beta J_{s(x)} \mid x_{0}=x, u_{0}=\gamma(x)\right] \\
& =J_{x}(\gamma)
\end{aligned}
$$

Thus $T^{k} W(x) \leq J_{x}(\gamma)$ for every $k \in \mathbb{N}$. Then, since the sequence $\left\{T^{k} W\right\}_{k \geq 0}$ converges to $V^{*}$ (again, by Banach's fixed point theorem), we have $T^{k} W(x) \rightarrow V^{*}(x)$ as $n \rightarrow \infty$. Thus, we have $V^{*}(x) \leq J_{x}(\gamma)$, as desired. 
Lemma 2.5: Suppose a policy $\pi^{*} \in \Pi$ satisfies the following equation for every $x \in \mathbb{X}$

$$
E\left[c\left(x, \pi^{*}(x)\right)\right]+\beta \sum_{y \in \mathbb{X}} P\left(y \mid x, \pi^{*}(x)\right) V^{*}(y)=\min _{u \in \mathbb{U}} E[c(x, u)]+\beta \sum_{y \in \mathbb{X}} P(y \mid x, u) V^{*}(y)
$$

then $\pi^{*}$ is an optimal policy.

The reader is referred to section 5.5 of [17] for this result. Indeed, the proof of Lemma 2.5 shows that $J_{x}\left(\pi^{*}\right)=V^{*}(x)$ for an optimal policy $\pi^{*}$ and any state $x$.

Lemma 2.6:

Let $\pi \in \Pi$ be a policy for which there exists $x \in \mathbb{X}$ such that

$$
E[c(x, \pi(x))]+\beta \sum_{y \in \mathbb{X}} P(y \mid, x, \pi(x)) V^{*}(y)>\min _{u \in \mathbb{U}} E[c(x, u)]+\beta \sum_{y \in \mathbb{X}} P(y \mid, x, u) V^{*}(y)
$$

Then $\pi \notin \Pi^{*}$.

Proof of lemma 2.6: Let $x_{0}$ be such a state. Then

$$
\begin{aligned}
J_{x_{0}}(\pi) & =E\left[c\left(x_{0}, \pi\left(x_{0}\right)\right)+\beta J_{s\left(x_{0}\right)}(\pi) \mid x_{0}, \pi\left(x_{0}\right)\right] \\
& \geq E\left[c\left(x_{0}, \pi\left(x_{0}\right)+\beta V^{*}\left(s\left(x_{0}\right)\right) \mid x_{0}, \pi\left(x_{0}\right)\right]\right. \\
& >\min _{u \in \mathbb{U}} E\left[c\left(x_{0}, u\right)+\beta V^{*}\left(s\left(x_{0}\right)\right) \mid x_{0}, u\right] \\
& =V^{*}\left(x_{0}\right) \\
& =J_{x_{0}}\left(\pi^{*}\right)
\end{aligned}
$$

Where the first inequality holds by Lemma 2.4, the strict inequality holds by our assumption on $\pi$, and $\pi^{*}$ is some optimal policy (whose existence was guaranteed by proposition 2.1), and lemma 2.5 justifies the final equality.) 
Proof of Proposition 2.3: Lemmas 2.4 and 2.5 prove the backward direction $(\Leftarrow)$, and Lemma 2.6 proves the forward direction $(\Rightarrow)$

In summary, this section has one result about the existence of optimal solutions (Proposition 2.1), and one result about characterizing them in a useful way (Proposition 2.3). Moreover, these results show that when the transition kernel $P$ and the cost function $c$ are known, one can repeatedly apply $T$ to any initial vector $V_{0}$, and the sequence $\left\{T^{n} V_{0}\right\}$ will converge to the unique fixed point $V^{*}$, from which we can recover all optimal policies.

Definition: value iteration is an iterative method for finding an optimal deterministic policy for a MDP by repeatedly applying $T$ to an initial vector $V_{0} \in \mathbb{R}^{\mathbb{X}}$.

For more on value iteration and MDPs, see [6].

\section{$2.2 \quad$ Reinforcement Learning for MDPs}

We have seen that value iteration is both a way to recover an optimal deterministic policy, and also a way to verify optimality (using Prop 2.3). Value iteration, however, relies on access to both the transition kernel and to the cost function. In practice, we might not have access to one or both, so we need an alternative way to recover (or verify) the optimality of a policy. Reinforcement learning can be used to overcome such obstacles. Reinforcement learning algorithms are a class of methods that involve interacting with the environment and using the feedback information to learn how to play optimally. In this section, we discuss the Q-learning algorithm, but refer the reader to [11] for other reinforcement learning algorithms. 
Q-learning is a very well-studied learning algorithm. For the seminal paper and foundational results, see [14], [15], and [12]. An agent is made to maintain and update a vector of estimates, whose components are meant to capture the long-run cost/reward of a state-action pair.

The player interacts with the environment by actually playing the game at hand and observing the consequences of playing particular actions in particular states. The player observes immediately the cost incurred and the state transition that the system undergoes. In this way, the salient features of $c(\cdot, \cdot)$ and $P[\cdot, \mid \cdot, \cdot]$ are revealed without any a priori knowledge of the system and also without building an explicit model.

Q-learning has the following form:

1. initialize a vector $Q_{0} \in \mathbb{R}^{\mathbb{X} \times \mathbb{U}}$

2. at time $t$, observe the current state $x_{t}$, and select an action $u_{t}$.

3. after playing the state-action pair $\left(x_{t}, u_{t}\right)$, observe the state transition (i.e. see $\left.x_{t+1}\right)$ and the cost incurred, (i.e. $\left.c\left(x_{t}, u_{t}\right)\right)$

4. update Q-factors, i.e. set $Q_{t+1}$.

5. return to step 2 .

The Q-factor update has the following form:

$$
Q_{t+1}(x, u)= \begin{cases}{\left[1-\alpha_{t}(x, u)\right] Q_{t}(x, u)+} & \\ \alpha_{t}(x, u)\left[c(x, u)+\beta \min _{v \in \mathbb{U}} Q_{t}\left(x_{t+1}, v\right)\right], & \text { if }\left(x_{t}, u_{t}\right)=(x, u) \\ Q_{t}(x, u) & \text { otherwise }\end{cases}
$$

where $\alpha_{t}(x, u) \in[0,1]$ is called the step-size or learning-rate. 
The Q-learning algorithm was shown to be a stochastic approximation algorithm in [12], and the Q-factor update was shown to be a noisy implementation of the mapping $F: \mathbb{R}^{\mathbb{X} \times \mathbb{U}} \rightarrow \mathbb{R}^{\mathbb{X} \times \mathbb{U}}$, whose components are given by:

$$
F Q(x, u)=E\left[c(x, u)+\beta \min _{v \in \mathbb{U}} Q(s(x), v) \mid x, u\right]
$$

This mapping $F$ is a contraction with respect to the infinity norm and thus has a unique fixed point $Q^{*}$. (The proof mimics that of lemma 2.2, and for that reason is omitted.) Furthermore, under appropriate conditions, we have that $Q_{t} \rightarrow Q^{*}$ as $t \rightarrow \infty$. The conditions include (i) the environment must be stationary (cost realizations and state transitions must not depend on time or external influences), (ii) each state-action pair must be played infinitely many times, and (iii) the step-sizes must meet requirements common for stochastic approximation algorithms. Namely, for every $(x, u) \in \mathbb{X} \times \mathbb{U}$, we have $\sum_{t} \alpha_{t}(x, u)=+\infty$ and $\sum_{t}\left(\alpha_{t}(x, u)\right)^{2}<\infty$. For a full treatment of standard Q-learning, see [12].

Lemma 2.7: Construct $V^{*} \in \mathbb{R}^{|\mathbb{X}|}$ as follows:

$$
V^{*}(x)=\min _{u \in \mathbb{U}} Q^{*}(x, u)
$$

Then $T V^{*}=V^{*}$ 
Proof of lemma 2.7:

$$
\begin{aligned}
T V^{*}(x) & =\min _{u \in \mathbb{U}} E\left[c(x, u)+\beta V^{*}(s(x)) \mid x, u\right] \\
& =\min _{u \in \mathbb{U}} E\left[c(x, u)+\beta \min _{w \in \mathbb{U}} Q^{*}(s(x), w) \mid x, u\right] \\
& =\min _{u \in \mathbb{U}} F Q^{*}(x, u) \\
& =\min _{u \in \mathbb{U}} Q^{*}(x, u) \\
& =V^{*}(x)
\end{aligned}
$$

Lemma 2.7 tells us that now we can use our previous results about value iteration and the value function $\left(V^{*}\right)$ without the DM knowing the cost function or the transition probabilities. In particular, we can construct and verify the optimality of policies if we have $Q^{*}$. 


\subsection{Stochastic Games}

Definition: A finite, discounted stochastic game has the following components:

- $N \in \mathbb{N}$ decision makers (also called players) labelled $1, \ldots, N$.

- A finite set of states $\mathbb{X}$

- For each player $i$, a set of control actions $\mathbb{U}^{i}$

- for each player $i$, a cost function $c^{i}: \mathbb{X} \times \mathbb{U}^{1} \times \cdots \times \mathbb{U}^{N} \rightarrow \mathbb{R}$

- A probability transition kernel $P\left[\cdot \mid x, u^{1}, \ldots, u^{N}\right]$ that determines the next state.

- For each player $i$, a discount factor $\beta^{i} \in(0,1)$.

We now provide some definitions that are analogous to those given for the MDP. Let us denote by $\mathcal{F}_{t}^{i}$ the $\sigma$-algebra generated by all of the information available to player $i$ at time $t$.

Definition: A policy $\gamma^{j}=\left(\gamma_{t}^{j}\right)_{t \geq 0}$ for player $j$ is admissible if for every $t \geq 0$ (i) $\gamma_{t}^{j}$ is $\mathcal{F}_{t}^{j}$-measurable, and (ii) $\operatorname{Im}\left(\gamma_{t}^{j}\right) \subseteq \mathcal{P}\left(\mathbb{U}^{j}\right)$, the set of probability measures on $\mathbb{U}^{j}$.

Let us introduce notation for important sets of policies:

$$
\begin{aligned}
& \Delta^{i}:=\left\{\gamma^{i}: \gamma^{i} \text { is admissible for player } i\right\} \\
& \Pi^{i}:=\left\{\gamma^{i}: \mathbb{X} \rightarrow \mathbb{U}^{i}\right\} \\
& \Delta:=\Delta^{1} \times \cdots \times \Delta^{N} \\
& \Pi:=\Pi^{1} \times \cdots \Pi^{N}
\end{aligned}
$$

Definition: $\gamma \in \Delta$ is called a joint policy. $\gamma=\left(\gamma^{1}, \ldots, \gamma^{N}\right)$, but we will sometimes write $\gamma=\left(\gamma^{i}, \gamma^{-i}\right)$ to isolate the policy of player $i$. Then $\gamma^{-i}=\left(\gamma^{1}, \ldots, \gamma^{i-1}, \gamma^{i+1}, \ldots, \gamma^{N}\right)$ Here, $-i$ stands to mean all agents except $i$ This convention for $-i$ is common in the literature, and will be used in other contexts, e.g. $\Pi^{-i}$ or $u^{-i}$. 


\section{Best responding and Equilibrium}

As in the single-agent case, players are interested in selecting an admissible policy to minimize their (long-run) cost function. However, now there is the added consideration of how other agents impact one's costs.

Fix $\gamma \in \Delta$. For each $x \in \mathbb{X}$, we define player $i$ 's long-term cost function as follows:

$$
J_{x}^{i}(\gamma)=E_{\gamma}\left[\sum_{k \geq 0} \beta^{i} c^{i}\left(x_{k}, u_{k}^{i}, u_{k}^{-i}\right) \mid x_{0}=x\right]
$$

where the expectation $E_{\gamma}[\cdot]$ is the conditional expectation given that actions are selected according to the admissible joint policy $\gamma$.

Definition: Let $\pi^{-i} \in \Delta^{-i}$. We say that a policy $\pi_{B R}^{i} \in \Delta^{i}$ is a best response to $\pi^{-i}$ if $J_{x}^{i}\left(\pi_{B R}^{i}, \pi^{-i}\right)=\inf \left\{J_{x}^{i}\left(\gamma^{i}, \pi^{-i}\right): \gamma^{i} \in \Delta^{i}\right\}$ for every state $x \in \mathbb{X}$. We will denote the set of deterministic best responses to a policy $\pi^{-i}$ by

$$
B R_{\pi^{-i}}^{i}=\left\{\pi^{* i} \in \Pi^{i}: J_{x}^{i}\left(\pi^{* i}, \pi^{-i}\right)=\inf \left\{J_{x}^{i}\left(\gamma^{i}, \pi^{-i}\right): \gamma^{i} \in \Delta^{i}\right\} \text { for every state } \mathrm{x} \in \mathbb{X}\right\}
$$

Definition: Let $\pi=\left(\pi^{i}, \pi^{-i}\right) \in \Delta$. We say that $\pi$ is an equilibrium joint policy if $\pi^{i}$ is a best response to $\pi^{-i}$ for every player $i$. This is called person-by-person optimality in team theory [18].

Notation: let $\Pi_{e q}:=\{\pi \in \Pi: \pi$ is an equilibrium $\}$ denote the set of deterministic equilibrium policies. Note: this set can be empty in general.

Definition: Let $\left(\pi^{i}, \pi^{-i}\right) \in \Delta$ be a policy. If $\tilde{\pi}^{i} \in B R_{\pi^{-i}}^{i}$ and there is some state $x \in \mathbb{X}$ such that $J_{x}^{i}\left(\pi^{i}, \pi^{-i}\right)>J_{x}^{i}\left(\tilde{\pi}^{i}, \pi^{-i}\right)$, then we say that $\tilde{\pi}^{i}$ is a strict best response w.r.t. 
$\left(\pi^{i}, \pi^{-i}\right)$

Definition: A (possibly finite) sequence $\left(\pi_{k}\right)_{k \geq 0}$, with $\pi_{k} \in \Pi$ for all $k$, is said to be a strict best response path if for every $k$, we have (i) there exists exactly one player $j$ for whom $\pi_{k+1}^{j} \neq \pi_{k}^{j}$, and (ii) $\pi_{k+1}^{j}$ is a strict best response with respect to $\pi_{k}$.

Definition: a discounted stochastic game is called weakly acyclic under strict best responses if there exists a strict best response path starting at each deterministic joint policy and ending at a deterministic equilibrium policy.

Definition: a stochastic game in which $c^{i} \equiv c$ and $\beta^{i} \equiv \beta$ is called a stochastic team game. Consequently, $J_{x}^{i}=J_{x}^{l}$ for all players $i, l$. To be explicit, we will use the notation $J_{x}=J_{x}^{i}$ when in the team setting.

Remark: discounted stochastic team games are weakly acyclic under strict best responses. Team problems are an important special case of weakly acyclic games.

Definition: in a stochastic team game, a joint policy $\pi \in \Delta$ is said to be team optimal if $J_{x}(\pi)=\inf _{\gamma \in \Delta} J_{x}(\gamma)$ for every state $x \in \mathbb{X}$.

\section{Claim 2.8:}

For any stochastic team game $G$, there exists a stationary deterministic joint policy that is team optimal, i.e.

$\exists \pi^{*} \in \Pi: J_{x}\left(\pi^{*}\right)=\inf _{\gamma \in \Delta} J_{x}(\gamma)$ for every $x \in \mathbb{X}$

Proof: one can define a Markov decision process out of the stochastic team game by defining the controller's action set to be the Cartesian product of all agents' action sets; then, an admissible joint policy is an admissible policy in this new MDP, and so 
proposition 2.1 is invoked to prove the claim $\square$.

Notation: we will denote the set of deterministic team optimal policies by $\Pi^{O P T}:=\left\{\pi^{*} \in \Pi: J_{x}\left(\pi^{*}\right)=\inf _{\gamma \in \Delta} J_{x}(\gamma)\right.$ for all $\left.x \in \mathbb{X}\right\}$. Note that this notion is only defined in the context of team games, and thus by Claim 2.8, $\Pi^{O P T} \neq \varnothing$.

Defining equilibrium by holding constant the policies of other agents is natural, especially in the context of games where players potentially have different, even opposing objectives. In such settings, a unilateral change in an individual's policy is all one can do to improve their standing. However, this definition is still sensible in the context of games where players have identical objectives, but it has one curious consequence: not all equilibrium policies are optimal in stochastic team games.

Fact 2.9: for a stochastic team game, we have

$$
\varnothing \neq \Pi^{O P T} \subseteq \Pi_{e q}
$$

And in general, the inclusion is proper.

\section{Characterizing Best Responses with Q-factors}

The notion of an equilibrium was defined while holding fixed the policies of all players except $i$. Let us probe that further.

Proposition 2.10:

For fixed stationary joint policy $\pi^{-i} \in \Delta^{-i}$, player $i$ faces a Markov Decision Process.

Proof: one constructs a new transition kernel $\tilde{P}$ by setting $\tilde{P}\left[y \mid x, u^{i}\right]:=P\left[y, \mid x,\left(u^{i}, \pi^{-i}(x)\right)\right]$ 
for every $x, y$ and $u^{i}$, and similarly uses $c$ to define a new cost function $\tilde{c}$

In view of Proposition 2.10, we can characterize an agent's set of deterministic best responses using the vector $Q_{\pi^{-i}}^{* i}$, the fixed point of the Q-learning process outlined above in section 2.2 .

Claim 2.11:

Let $\gamma^{-i} \in \Delta^{-i}$ be stationary. Then,

$$
B R_{\gamma^{-i}}^{i}=\left\{\pi^{i} \in \Pi^{i}: Q_{\pi^{-i}}^{* i}\left(x, \pi^{i}(x)\right)=\min _{u^{i} \in \mathbb{U}^{i}} Q_{\pi^{-i}}^{* i}\left(x, u^{i}\right)\right\}
$$

In general, an agent may have no deterministic best responses. That is, $B R_{\gamma^{-i}}^{i}=\varnothing$ may be the case. Proposition 2.10 (and its implication, Claim 2.11) inform us that the set is non-empty when $\gamma^{-i}$ is stationary.

\subsection{Literature Review}

Now that we have introduced the necessary notions and notation, we can survey the existing literature on methods for coordinating agents in a decentralized setting.

In [9], the authors do a simulation study of standard Q-learning applied to a 2-person team game. The goal in that paper was to replace communication by learning. The algorithms implemented did not make use of shared information or prior knowledge about the model, but also did not provide any rigorous results.

In [3], the authors consider two Q-learning based reinforcement learning algorithms for use in repeated team games (stochastic team games with $|\mathbb{X}|=1$ ). That work 
claims that equilibrium will be found with arbitrarily high probability, but no proof is given. The repeated game setting is a special case of stochastic games, and the joint action learner considered there requires considerably greater shared information than we wish to use.

Outside of the realm of Q-learning generalizations, [13] presents an algorithm based on adaptive play (see [16]) and proves convergence to a team optimal equilibrium. Given their objectives, this work is notably successful and rigorous compared to what came before it. However, the method uses significantly greater shared information, as it requires knowledge of both other players and their actions, and is not applicable to our setting.

Further rigorous results can be found in [7] and [2], noting again that their settings and ours are different.

In [8], the authors provide three learning algorithms, to be applied in increasingly more general contexts. They prove convergence, in some sense, to optimal play in a repeated team game. They further establish a convergence result for the class of weakly acyclic games-a step in the direction of greater generality. The algorithms presented are individually rational and do not suffer from informational and communication sharing problems, but the setting is more narrow than the setting we consider here.

In [1], the authors provide a meaningful generalization of standard Q-learning-called Decentralized Q-learning-in which agents experiment slowly, and the stationary environment necessary for Q-learning to converge is well-approximated. (This addresses the problem of teaching while learning in multi-agent set-ups, mentioned in [10].) The 
learning rule there will lead to an equilibrium joint policy in self-play, however fact 2.9 underscores a shortcoming of this guarantee: an equilibrium joint policy may not be team optimal, and indeed can be arbitrarily worse.

The contribution in this thesis builds on [1]. Most notably, the notion of an exploration phase is borrowed, and the learning algorithm within a single exploration phase is identical to their algorithm. The information processing and policy update between exploration phases is where their method and the present method diverge. 


\section{Chapter 3}

\section{Main Results}

\section{Chapter Outline}

This chapter contains the main contribution of this thesis. We present a learning algorithm, state a convergence theorem, and provide a proof. The chapter is divided as follows:

In section 3.1, Algorithm 1 from [1] and our Algorithm B are presented in pseudocode. For reference, we also state the main result from [1], and compare that result with the present objective.

In section 3.2 , we formally state the theorem we will prove.

In section 3.3, we provide an overview of the proof method. This section justifies the sections full of supporting results, namely sections 3.4, 3.5, and 3.6.

Finally, the proof of the theorem is given in section 3.7 
We now present Algorithm 1 from [1]. This algorithm can be implemented for discounted stochastic games, using the notation specified on page 15 .

\subsection{The Algorithms in Pseudocode}

\section{Algorithm 1 from [1] (for $D M^{i}$ )}

Set Parameters

- $\mathbb{Q}^{i}$ a compact subset of $\mathbb{R}^{\mathbb{X} \times \mathbb{U}^{i}}$, where $\left|\mathbb{X} \times \mathbb{U}^{i}\right|$ is the number of pairs $\left(x, u^{i}\right)$.

- A sequence of positive integers $\left\{T_{k}\right\}_{k \geq 0}$, the exploration phase lengths.

- Define $t_{0}=0$, and in general $t_{k+1}=t_{k}+T_{k}$.

- $\rho^{i} \in(0,1)$ an experimentation probability

- $\lambda^{i} \in(0,1)$ the probability of inertia

- $\delta^{i}>0$ a tolerance for suboptimality

- $\left\{\alpha_{n}^{i}\right\}_{n \geq 0}$ a sequence of step sizes such that

$\alpha_{n}^{i} \in[0,1] \quad \sum_{n \geq 0} \alpha_{n}^{i}=\infty \quad \sum_{n \geq 0}\left(\alpha_{n}^{i}\right)^{2}<\infty$

(e.g. $a_{n}^{i}=1 / n^{r}$, for $r \in(1 / 2,1]$.)

- Select arbitrary $\pi_{0}^{i} \in \Pi^{i}$ and $Q_{0} \in \mathbb{Q}^{i}$,

- Receive the initial stage $x_{0}$

Iterate $k \geq 0$ ( $k^{t h}$ exploration phase)

Iterate $t=t_{k}, t_{k}+1, \ldots, t_{k+1}-1$

$$
u_{t}^{i}=\left\{\begin{array}{l}
\pi_{k}^{i}\left(x_{t}\right) \text { w.p. } 1-\rho^{i} \\
\text { any } u^{i} \in \mathbb{U}^{i} \text { w.p. } \rho^{i} / \mathbb{U}^{i}
\end{array}\right.
$$

Receive cost $c^{i}\left(x_{t}, u_{t}^{i}, u_{t}^{-i}\right)$

Receive the next state $x_{t+1}$ (chosen according to $P\left[\cdot \mid x_{t}, u_{t}^{i}, u_{t}^{-i}\right]$ ) $n_{t}^{i}=$ the number of visits to $\left(x_{t}, u_{t}^{i}\right)$ in the $k^{t h}$ exploration phase (up to and including $t$ )

$$
\begin{aligned}
& Q_{t+1}^{i}\left(x_{t}, u_{t}^{i}\right)=\left(1-\alpha_{n_{t}^{i}}^{i}\right) Q_{t}^{i}\left(x_{t}, u_{t}^{i}\right) \\
& \quad+\alpha_{n_{t}^{i}}^{i}\left[c^{i}\left(x_{t}, u_{t}^{i}, u_{t}^{-i}\right)+\beta^{i} \min _{v^{i} \in \mathbb{U}^{i}} Q_{t}^{i}\left(x_{t+1}, v^{i}\right)\right]
\end{aligned}
$$




$$
Q_{t+1}^{i}\left(x, u^{i}\right)=Q_{t}^{i}\left(x, u^{i}\right) \text { for every }\left(x, u^{i}\right) \neq\left(x_{t}, u_{t}^{i}\right)
$$

End

$$
\begin{aligned}
& B R_{k}^{i}=\left\{\hat{\pi}^{i} \in \Pi^{i}: Q_{t_{k+1}}^{i}\left(x, \hat{\pi}^{i}(x)\right)\right. \\
& \left.\leq \min _{v^{i} \in \mathbb{U}^{i}} Q_{t_{k+1}}^{i}\left(x, v^{i}\right)+\delta^{i} \text { for every } x \in \mathbb{X}\right\} \\
& \text { If } \pi_{k}^{i} \in B R_{k}^{i} \text {, set } \pi_{k+1}^{i}=\pi_{k}^{i} \text {. }
\end{aligned}
$$

Else

$$
\begin{aligned}
& \qquad \pi_{k+1}^{i}=\left\{\begin{array}{l}
\pi_{k}^{i} \text { w.p. } \lambda^{i} \\
\text { any } \pi^{i} \in B R_{k}^{i} \text { w.p. }\left(1-\lambda^{i}\right) /\left|B R_{k}^{i}\right|
\end{array}\right. \\
& \text { End } \begin{array}{l}
\text { Reset } Q_{t_{k+1}}^{i} \text { to any } Q^{i} \in \mathbb{Q}^{i} \text {, e.g. project } Q_{t_{k+1}}^{i} \text { onto } \mathbb{Q}^{i}
\end{array}
\end{aligned}
$$

End 


\section{Main Theorem from [1]}

Assumption 1: For all $\left(x, x^{\prime}\right) \in \mathbb{X} \times \mathbb{X}$, there exists a finite integer $H \geq 0$ and joint decisions $\tilde{u}_{0}, \tilde{u}_{1}, \ldots, \tilde{u}_{H}$ such that

$$
P\left[x_{H+1}=x^{\prime} \mid x_{0}=x, u_{0}=\tilde{u}_{0}, u_{1}=\tilde{u}_{1}, \ldots, u_{H}=\tilde{u}_{H}\right]>0
$$

Assumption 2: For all $i, 0<\delta^{i}<\bar{\delta}$ and $0<\rho^{i}<\bar{\rho}$, where $\bar{\delta}$ and $\bar{\rho}$ (which depend only on the parameters of the game at hand) are defined in [1, Appendix B].

Theorem 1: Consider a discounted stochastic game that is weakly acyclic under strict best responses, as defined on page 17. Suppose that each $D M^{i}$ updates its policies by Algorithm 1. Let Assumption 1 and 2 hold.

(i) For any $\epsilon>0$ there exists $\tilde{T}<\infty, \tilde{k}<\infty$ such that if $\min _{j} T_{j} \geq \tilde{T}$ then

$$
P\left(\pi_{k} \in \Pi_{e q}\right) \geq 1-\epsilon, \text { for all } k \geq \tilde{k}
$$

(ii) if $\lim _{k \rightarrow \infty} T_{k}=\infty$, then $\lim _{k \rightarrow \infty} P\left(\pi_{k} \in \Pi_{e q}\right)=1$

(iii) There exists a sequence of finite integers $\left\{\tilde{T}_{k}\right\}_{k \geq 0}$ such that if $T_{k} \geq \tilde{T}_{k}$ for all $k$, then

$$
P\left(\pi_{k} \rightarrow \pi^{*}, \text { for some } \pi^{*} \in \Pi_{e q}\right)=1
$$

where $\Pi_{e q}$ is the set of equilibrium joint policies, defined on 16 . 


\section{Remarks and Goals}

Stochastic team games are weakly acyclic under strict best responses, and thus Algorithm 1's result applies in the stochastic team game setting. The generality of the result comes at a cost: as noted in fact 2.9, a person-by-person optimal equilibrium need not be a team optimal equilibrium (defined on page 17). In fact one can construct games in which a person-by-person optimal equilibrium is arbitrarily worse than optimal: consider this two-player repeated game with $\alpha>1$

\begin{tabular}{|c|c|c|}
\hline & Left & Right \\
\hline Up & $\alpha$ & $1+\alpha$ \\
\hline Down & $1+\alpha$ & 1 \\
\hline
\end{tabular}

Playing (Up, Left) in each period is person-by-person optimal, since unilaterally deviating from this policy increases the cost incurred by 1 in that period. Playing (Down, Right) in every period would reduce cost to only 1 in each period. (Up, Left) yields a long-term cost of $\alpha /(1-\beta)$, while (Down, Right) yields a long-term cost of $1 /(1-\beta)$. The ratio, $\alpha$, can be made arbitrarily large. Indeed, for $\alpha$ large, the use of random policies outperforms this person-by-person optimal equilibrium.

Self-play using Algorithm 1 might then lead to convergence to a rather unfavourable joint policy (e.g. (Up, Left) in the example above). This should not be taken as a criticism of [1]: their work applies to a wider class of games, and the notion of team optimality (generalized to social optimality, perhaps) does not have a natural candidate in many weakly acyclic games. With that said, our first objective here is to provide a learning rule, specifically for use in stochastic team games, that converges not just to equilibrium, but to the team optimal equilibrium. 
After good performance with respect to long-run cost, a second objective is to design a learning rule with the desirable attributes mentioned in [1], namely the use of only locally available information, no communication between players during play, and individual rationality. In this context, individual rationality requires that players act in their own best interest. In particular, if knowledge of the model is limited, players might not know that they are playing a stochastic team game, and employing unselfish, equilibrium-seeking behaviour is hard to justify.

The final remark to make is about the notion of convergence used in [1]: the result is about convergence in baseline policies, there labeled $\pi_{k}$. As mentioned in the section 2.2, Q-learning requires visiting all state-action pairs infinitely often. What this means for a Q-learning in a multi-agent setting is that any player must occasionally play actions different from the policy's prescription. Algorithm 1 explicitly programs in such exploration. Action selection that exploits previous learning is possible (see Boltzmann action selection, discussed in [1], [3], and elsewhere), for example, but even when such a strategy is used, the probability of playing non-policy actions is positive.

In this thesis, we continue in the tradition of [1], accepting as a consequence that some off-policy play is needed in order to explore and exploit simultaneously. If players coordinate on an optimal baseline policy and also experiment with low probability, then the stage game outcomes will coincide with optimal play with high probability. One might hope that the probability of the coincidence of actual and optimal gameplay should tend to 1 , but we leave that for future work. In any case, the subject of evaluating the performance of a learning algorithm remains an interesting one. 
Next, we present our new learning algorithm. It is designed for stochastic team games, were $c^{i} \equiv c$, but is presented here for general stochastic game:

\section{Algorithm B (for $D M^{i}$ )}

Set Parameters

- $\mathbb{Q}^{i}$ a compact subset of $\mathbb{R}^{\left|\mathbb{X} \times \mathbb{U}^{i}\right|}$

- A sequence of positive integers $\left\{T_{k}\right\}_{k \geq 0}$, the exploration phase lengths.

- Define $t_{0}=0$, and in general $t_{k+1}=t_{k}+T_{k}$.

- $\rho^{i} \in(0,1)$ an experimentation probability

- $\lambda^{i} \in(0,1)$ the probability of inertia

- $\delta^{i}>0$ a tolerance for suboptimality when constructing best-response sets

- $d>0$ a tolerance for sub-optimality when evaluating a baseline policy (common for every player)

- $\left\{\alpha_{n}^{i}\right\}_{n \geq 0}$ a sequence of step sizes such that

$$
\begin{aligned}
& \alpha_{n}^{i} \in[0,1] \quad \sum_{n \geq 0} \alpha_{n}^{i}=\infty \quad \sum_{n \geq 0}\left(\alpha_{n}^{i}\right)^{2}<\infty \\
& \text { (e.g. } \left.a_{n}^{i}=1 / n^{r}, \text { for } r \in(1 / 2,1] .\right)
\end{aligned}
$$

- Common $\gamma_{1}, \gamma_{2} \in(0,1)$, probabilities for randomly updating baseline policy

- $W \in \mathbb{N}$, a window size

- Select arbitrary $\pi_{0}^{i} \in \Pi^{i}$ and $Q_{0} \in \mathbb{Q}^{i}$

- Receive the initial stage $x_{0}$

Iterate $k \geq 0$ ( $k^{\text {th }}$ exploration phase)

Iterate $t=t_{k}, t_{k}+1, \ldots, t_{k+1}-1$

$$
u_{t}^{i}=\left\{\begin{array}{l}
\pi_{k}^{i}\left(x_{t}\right) \text { w.p. } 1-\rho^{i} \\
\text { any } u^{i} \in \mathbb{U}^{i} \text { w.p. } \rho^{i} / \mathbb{U}^{i}
\end{array}\right.
$$

Receive cost $c^{i}\left(x_{t}, u_{t}^{i}, u_{t}^{-i}\right)$

Receive the next state $x_{t+1}$ (chosen according to $P\left[\cdot \mid x_{t}, u_{t}^{i}, u_{t}^{-i}\right]$ ) $n_{t}^{i}=$ the number of visits to $\left(x_{t}, u_{t}^{i}\right)$ in the $k^{t h}$ exploration phase 
(up to and including $t$ )

$$
\begin{aligned}
& Q_{t+1}^{i}\left(x_{t}, u_{t}^{i}\right)=\left(1-\alpha_{n_{t}^{i}}^{i}\right) Q_{t}^{i}\left(x_{t}, u_{t}^{i}\right) \\
& +\alpha_{n_{t}^{i}}^{i}\left[c^{i}\left(x_{t}, u_{t}^{i}, u_{t}^{-i}\right)+\beta^{i} \min _{v^{i} \in \mathbb{U}^{i}} Q_{t}^{i}\left(x_{t+1}, v^{i}\right)\right] \\
& Q_{t+1}^{i}\left(x, u^{i}\right)=Q_{t}^{i}\left(x, u^{i}\right) \text { for every }\left(x, u^{i}\right) \neq\left(x_{t}, u_{t}^{i}\right)
\end{aligned}
$$

End

$$
\begin{aligned}
& B R_{k}^{i}=\left\{\hat{\pi}^{i} \in \Pi^{i}: Q_{t_{k+1}}^{i}\left(x, \hat{\pi}^{i}\right)\right. \\
& \left.\quad \leq \min _{v^{i} \in \mathbb{U}^{i}} Q_{t_{k+1}}^{i}\left(x, v^{i}\right)+\delta^{i} \text { for every } x \in \mathbb{X}\right\} \\
& S_{k}^{i}=\sum_{x \in \mathbb{X}} Q_{t_{k+1}}^{i}\left(x, \pi_{k}^{i}(x)\right)
\end{aligned}
$$

If $k \in\{0,1, \ldots, W-1\}$, select $\pi_{k+1}^{i}$ uniformly at random.

Else for some $B \geq 1$, we have $k \in\{B \cdot W, B \cdot W+1, \ldots,(B+1) \cdot W-1\}$

$$
\begin{aligned}
& \Lambda_{B-1}^{i}=\min \left\{S_{(B-1) W}^{i}, S_{(B-1) W+1}^{i}, \ldots, S_{B W-1}^{i}\right\} \\
& \text { If } S_{k}^{i}<\Lambda_{B-1}^{i}+d
\end{aligned}
$$

Select $\pi_{k+1}^{i}=$ any $\pi \in \Pi^{i}$ w.p. $\gamma_{1} /\left|\Pi^{i}\right|$

w.p. $1-\gamma_{1}$, select $\pi_{k+1}^{i}$ according to "if $\left(\pi_{k}^{i} \in B R_{k}^{i}\right.$, set $\left.\pi_{k+1}^{i}=\pi_{k}^{i}\right)$, else $\left(\pi_{k+1}^{i}=\pi_{k}^{i}\right.$ w.p. $\lambda^{i}$ and $\pi_{k+1}^{i}=$ any $\pi^{i} \in B R_{k}^{i}$ w.p. $\left.\left(1-\lambda^{i}\right) /\left|B R_{k}^{i}\right|\right) "$ Else $\left(S_{k}^{i} \geq \Lambda_{B-1}^{i}+d\right)$

Select $\pi_{k+1}^{i}=$ any $\pi \in \Pi^{i}$ w.p. $\left(\gamma_{1}+\gamma_{2}\right) /\left|\Pi^{i}\right|$

w.p. $1-\left(\gamma_{1}+\gamma_{2}\right)$, select $\pi_{k+1}^{i}$ according to "if $\left(\pi_{k}^{i} \in B R_{k}^{i}\right.$, set $\pi_{k+1}^{i}=$ $\left.\pi_{k}^{i}\right)$, else $\left(\pi_{k+1}^{i}=\pi_{k}^{i}\right.$ w.p. $\quad \lambda^{i}$ and $\pi_{k+1}^{i}=$ any $\pi^{i} \in B R_{k}^{i}$ w.p. $\quad(1-$ $\left.\left.\lambda^{i}\right) /\left|B R_{k}^{i}\right|\right)^{\prime}$

End

End

Reset $Q_{t_{k+1}}^{i}$ to any $Q^{i} \in \mathbb{Q}^{i}$ (e.g. project $Q_{t_{k+1}}^{i}$ onto $Q^{i} \in \mathbb{Q}^{i}$ )

End $k^{t h}$ exploration phase. (and continue to exploration phase $k+1$ ) 


\subsection{Theorem Statement}

Consider a finite, discounted stochastic team game, and let $\Pi^{O P T}$ denote the set of team optimal joint policies.

Assumption 1: For any $x, x^{\prime} \in \mathbb{X}$, there exists $H \in \mathbb{N}$ and joint actions $\tilde{u}_{0}, \ldots, \tilde{u}_{H}$ such that

$$
P\left(x_{H+1}=x^{\prime} \mid x_{0}=x, u_{j}=\tilde{u}_{j} \text { for every } j \in\{0,1, \ldots, H\}\right)>0
$$

Consider a stochastic team game in which all players use Algorithm $\mathrm{B}$.

Let $\epsilon>0$. Let assumption 1 hold. There exist $\gamma_{1}^{*}, \gamma_{2}^{*}, \tilde{W}, L, \bar{\rho}, \bar{\delta}, d^{*}$ (all depending on $\epsilon$ ) such that if

- $\gamma_{1}=\gamma_{1}^{*}$,

- $\gamma_{2}=\gamma_{2}^{*}$,

- $W=\tilde{W}$,

- $d=d^{*}$,

- $T_{k} \geq L$ for every $k$,

- $\rho^{i} \in(0, \bar{\rho})$ for every $i$,

- $\delta^{i} \in(0, \bar{\delta})$ for every $i$,

then there exists $M \in \mathbb{N}$ such that

$$
\operatorname{Prob}\left(\pi_{n} \in \Pi^{O P T}\right) \geq 1-\epsilon \text { for every } n \geq M
$$




\subsection{Proof Overview}

\section{Intuition}

Before giving an overview of the proof, let us observe the three types of baseline policy updates that this algorithm suggests:

Firstly, players can switch to a strict best response. This update type is individually rational, and in general leads to better system performance. We can think of it as analogous to gradient descent. But as with gradient descent, it can lead to being stuck in a local optimum that is globally suboptimal.

Secondly, players can use the inertial response of doing nothing. This update type was used in [1] to replace the communication needed for agreeing on the order in which players will update their policies. Note that without inertia (or an agreed upon order for updating), curious pathologies may appear. For example, consider a repeated game with two players (named 1 and 2) and two actions (called a and b) in which the players are rewarded for playing the same action. Suppose 1's initial policy is to always play a, and 2's initial policy is to always play b. Then, if players best responded without inertia, then after the switch player 1 would always play b, while player 2 would always play a. That is, we would enter a cycle of coordination failures.

Finally, the last update type is switching to a random new policy. On its face, this does not seem like an individually rational suggestion, but the technique of introducing noise to escape local optima is an established practice in optimization (for example, see [5]). In keeping with such practices, our learning algorithm was constructed such 
that the likelihood of a random update decreases as the quality of the equilibrium increases.

In the ideal case, players would mostly follow strict best responding with inertia (so they would arrive at an equilibrium in a more direct fashion than if they only updated their policy at random) and they would occasionally randomize when they were sure they could do better, to avoid becoming stuck in a local equilibrium.

If the players were able to tell with certainty whether they were playing an optimal policy, the problem would be trivial. Nevertheless, the ideal case suggests to us a second process that performs slightly worse but is analytically tractable and much easier to (approximately) implement. In this second ideal case, players would mostly follow best responding with inertia, they would occasionally randomize when they were sure they could do better, but here they also would infrequently randomize when they think they are at an optimum.

If randomized updating is dramatically less likely (but still possible) when playing an optimal equilibrium, then we are dealing with an irreducible, aperiodic Markov chain on a finite set, and the chain will spend most of its time in the set of optimal joint policies. This is the motivation for designing the learning algorithm as we have.

\section{Proof Program}

In section 3.4, we consider a class of Markov chains to be used for comparison to our actual process. We start by defining a so-called oracle update rule, in which an oracle informs the players whether the joint policy is optimal or not, and then gives each player their current best response set. Players then use this information to update 
their policy using a mixture of best responding, randomizing, and inertia.

This (idealized) process of oracle revelation followed by policy updating induces a time homogenous Markov chain on the set of deterministic joint policies, П. The transition kernel depends on the game played and the parameters used by the players: $\gamma_{1}$ is the probability of randomly updating one's policy when already at an optimum. $\gamma_{2}$ is the added probability of randomly updating one's policy when not at an optimum. $\lambda^{i}$ is the probability of inertia for player $i$.

We denote the transition kernel $A^{\gamma_{1}, \gamma_{2}}$. The main result of the section is about $\mu_{\gamma_{1}, \gamma_{2}}^{*}$, the unique invariant measure of $A^{\gamma_{1}, \gamma_{2}}$. It states that after fixing $\gamma_{2}$,

$$
\lim _{\gamma_{1} \rightarrow 0^{+}} \sum_{\pi \in \Pi^{O P T}} \mu_{\gamma_{1}, \gamma_{2}}^{*}(\pi)=1
$$

In section 3.5, we state and prove some lemmas about so-called similar matrices. In particular, we investigate the following question: Suppose a (transition) matrix $J: \mathbb{R}^{n} \rightarrow \mathbb{R}^{n}$ has the property that $\lim _{k} \mu_{0} J^{k}=\mu^{*}$ for any initial (probability) vector $\mu_{0}$. Then, if $\left\{H_{k}\right\}_{k \geq 0}$ is a sequence of matrices that are "similar" to $J$ (in a way we will formalize) will the sequence $\left\{\mu_{0} H_{0} H_{1} \cdots H_{k}\right\}_{k \geq 0}$ behave like the sequence $\left\{\mu_{0} J^{k}\right\}_{k \geq 0}$ ? We provide conditions under which the answer to this question is affirmative. In particular, for arbitrary $\epsilon>0$, we provide conditions under which

$$
\limsup _{k \rightarrow \infty}\left\|\mu^{*}-\mu_{0} H_{0} H_{1} \cdots H_{k}\right\|_{1}<\epsilon
$$

Section 3.5's result pertains to our context as follows: if we denote the baseline policy in exploration phase $k$ by $\pi_{k}$, then we can define $\mu_{k}(\pi):=\operatorname{Prob}\left(\pi_{k}=\pi\right)$ for all joint 
policies $\pi \in \Pi$. If we further define $C_{k}\left(\pi, \pi^{\prime}\right):=\operatorname{Prob}\left(\pi_{k+1}=\pi^{\prime} \mid \pi_{k}=\pi\right)$ for every $\pi, \pi^{\prime} \in \Pi$, then by the law of total probability, we have $\mu_{k+1}=\mu_{k} C_{k}$. Our interest lies in $\liminf \inf _{n \rightarrow \infty} \operatorname{Prob}\left(\pi_{n} \in \Pi^{O P T}\right)$, i.e. $\operatorname{in~} \liminf _{n \rightarrow \infty} \sum_{\pi \in \Pi \text { OPT }} \mu_{n}(\pi)$. So, we are interested in a sequence $\left\{\mu_{0} C_{0} C_{1} \cdots C_{k}\right\}_{k \geq 0}$. The comparison Markov chains from section 3.4 will be used as an analog for $J$ in section 3.5, and the result from 3.4 and 3.5 will be used with the triangle inequality to argue that

$$
\liminf _{n \rightarrow \infty} \sum_{\pi \in \Pi^{O P T}} \mu_{0} C_{0} C_{1} \cdots C_{n}(\pi)>1-\epsilon
$$

In section 3.6, we put the pieces together and handle the details of the proof. We argue that appropriate parameter selection results in the matrix of conditional probabilities $C_{k}$ (analogous to $H_{k}$ 's) mimicking the oracle process $A^{\gamma_{1}, \gamma_{2}}$ (analogous to $J$ ) to any degree of similarity.

At this point we should stress something: the analysis in the proof does not use or make reference to the actual transition matrices, which are random variables (i.e., random stochastic matrices whose realization would depend on the past history). Instead, it makes reference to the conditional probabilities from one stage to another one, mapping the vectors of occupation probabilities from one stage to the next. (The reader is referred to 3.6 for an explicit description.) The analysis here approximates a non-Markovian system with a Markovian one, but the analysis does not ever use the operational transition kernels. As a consequence of our work, the transition kernels will behave like $A^{\gamma_{1}, \gamma_{2}}$ with high probability, however this fact is not relevant to the analysis.

In the section 3.7 , we complete the proof. 


\subsection{A Markov Chain to be Used for Comparison}

\section{Transition Matrix $A^{\gamma_{1}, \gamma_{2}}$}

Consider a time homogenous Markov chain on $\Pi$, where transitions are determined as follows:

- An oracle correctly tells player $i$ whether $\pi_{k} \in \Pi^{O P T}$ or not.

- If $\pi_{k} \in \Pi^{O P T}$, then player $i$ selects $\pi_{k+1}$ as follows:

$\pi_{k+1}=$ any $\pi^{i}$ with probability $\gamma_{1} /\left|\Pi^{i}\right|$

$\pi_{k+1}$ selected according to $\mathrm{BR}$ with inertia with probability $1-\gamma_{1}$

(BR with inertia described here:)

If $\pi_{k}^{i}$ is a best response to $\pi_{k}^{-i}$, then $\pi_{k+1}^{i}=\pi_{k}^{i}$

Else $\pi_{k}^{i}$ is not a best response to $\pi_{k}^{-i}$, then

$$
\pi_{k+1}^{i}=\left\{\begin{array}{l}
\pi_{k}^{i} \text { w.p. } \lambda^{i}>0(\text { inertia }) \\
\text { any best response } \pi^{i} \text { w.p }\left(1-\lambda^{i}\right) /(\# \text { best responses })
\end{array}\right.
$$

- Else $\left(\pi_{k} \notin \Pi^{O P T}\right)$ then player $i$ selects $\pi_{k+1}$ as follows:

$\pi_{k+1}=\pi^{i}$ with probability $\left(\gamma_{1}+\gamma_{2}\right) /\left|\Pi^{i}\right|$

$\pi_{k+1}$ selected according to BR with inertia with probability $1-\left(\gamma_{1}+\gamma_{2}\right)$

(Note: in this case, player $i$ has access to their correct best response set)

If $\pi_{k}^{i}$ is a best response to $\pi_{k}^{-i}$, then $\pi_{k+1}^{i}=\pi_{k}^{i}$

Else $\pi_{k}^{i}$ is not a best response to $\pi_{k}^{-i}$, then 


$$
\pi_{k+1}^{i}=\left\{\begin{array}{l}
\pi_{k}^{i} \text { w.p. } \lambda^{i}>0(\text { inertia }) \\
\text { any best response } \pi^{i} \text { w.p }\left(1-\lambda^{i}\right) /(\# \text { best responses })
\end{array}\right.
$$

For this Markov chain, there is no learning and no approximation: the player observes truthfully whether $\pi_{k}$ is a team optimal equilibrium, and observes their true best response set.

If $\gamma_{1}, \gamma_{2}>0$, we have an irreducible, aperiodic Markov chain on the finite set $\Pi$. We denote the transition kernel by $A_{\lambda}^{\gamma_{1}, \gamma_{2}}$. Indeed, $\lambda=\left(\lambda^{1}, \ldots, \lambda^{N}\right)$ influences the transition probabilities and cannot be neglected; however, for our purposes it has no impact (in that the lower bounds we derive are valid for any valid choice of $\lambda$ ) and will be dropped for notational ease. With this understanding, we continue with $A^{\gamma_{1}, \gamma_{2}}$.

Since $\Pi$ is finite and we have an aperiodic, irreducible chain, there exists a unique invariant probability measure. We denote by $\mu_{\gamma_{1}, \gamma_{2}, \lambda}^{*}$ the unique probability distribution on $\Pi$ such that

$$
\begin{aligned}
& \mu_{\gamma_{1}, \gamma_{2}, \lambda}^{*}=\mu_{\gamma_{1}, \gamma_{2}, \lambda}^{*} A^{\gamma_{1}, \gamma_{2}} \\
& \mu_{\gamma_{1}, \gamma_{2}, \lambda}^{*}(\pi) \geq 0 \quad \text { for every } \pi \in \Pi \\
& \sum_{\pi \in \Pi} \mu_{\gamma_{1}, \gamma_{2}, \lambda}^{*}(\pi)=1
\end{aligned}
$$

For ease of notation, let us proceed with $\mu^{*}=\mu_{\gamma_{1}, \gamma_{2}, \lambda}^{*}$, and return to the long-hand when necessary.

We can compute a lower bound on $\mu^{*}\left(\Pi^{O P T}\right)=\sum_{\pi \in \Pi^{O P T}} \mu^{*}(\pi)$ : 


$$
\begin{aligned}
\mu^{*}\left(\Pi^{O P T}\right) & =\sum_{\pi \in \Pi^{O P T}} \mu^{*}(\pi) \\
& =\sum_{\pi \in \Pi^{O P T}}\left(\sum_{a \in \Pi} \mu^{*}(a) A^{\gamma_{1}, \gamma_{2}}(a, \pi)\right) \quad\left(\mathrm{U} \operatorname{sing} \mu^{*}=\mu^{*} A^{\gamma_{1}, \gamma_{2}}\right) \\
& =\sum_{\pi \in \Pi^{O P T}}\left[\sum_{b \in \Pi^{O P T}} \mu^{*}(b) A^{\gamma_{1}, \gamma_{2}}(b, \pi)+\sum_{c \in \Pi \backslash \Pi^{O P T}} \mu^{*}(c) A^{\gamma_{1}, \gamma_{2}}(c, \pi)\right] \\
& =\sum_{\pi \in \Pi^{O P T}}\left[\sum_{b \in \Pi^{O P T}} \mu^{*}(b) A^{\gamma_{1}, \gamma_{2}}(b, \pi)\right]+\sum_{\pi \in \Pi \text { OPT }}\left[\sum_{c \in \Pi \backslash \Pi^{O P T}} \mu^{*}(c) A^{\gamma_{1}, \gamma_{2}}(c, \pi)\right] \\
& =\sum_{b \in \Pi^{O P T}}\left[\sum_{\pi \in \Pi O P T} \mu^{*}(b) A^{\gamma_{1}, \gamma_{2}}(b, \pi)\right]+\sum_{c \in \Pi \backslash \Pi O P T}\left[\sum_{\pi \in \Pi^{O P T}} \mu^{*}(c) A^{\gamma_{1}, \gamma_{2}}(c, \pi)\right] \\
& =\sum_{b \in \Pi^{O P T}} \mu^{*}(b)\left[\sum_{\pi \in \Pi^{O P T}} A^{\gamma_{1}, \gamma_{2}}(b, \pi)\right]+\sum_{c \in \Pi \backslash \Pi O P T} \mu^{*}(c)\left[\sum_{\pi \in \Pi^{O P T}} A^{\gamma_{1}, \gamma_{2}}(c, \pi)\right]
\end{aligned}
$$

The update rule implies the following useful inequalities hold (independent of $\lambda$, as mentioned):

$$
\begin{aligned}
\theta_{1} \in \Pi \backslash \Pi^{O P T}, \theta_{2} \in \Pi^{O P T} \Rightarrow A^{\gamma_{1}, \gamma_{2}}\left(\theta_{1}, \theta_{2}\right) \geq\left(\gamma_{1}+\gamma_{2}\right)^{N} /|\Pi| \\
\theta^{*}, \theta_{2} \in \Pi^{O P T}, \theta^{*} \neq \theta_{2} \Rightarrow A^{\gamma_{1}, \gamma_{2}}\left(\theta^{*}, \theta_{2}\right) \geq \gamma_{1}{ }^{N} /|\Pi| \\
\theta^{*} \in \Pi^{O P T} \Rightarrow A^{\gamma_{1}, \gamma_{2}}\left(\theta^{*}, \theta^{*}\right) \geq\left(1-\gamma_{1}\right)^{N}+\gamma_{1}^{N} /|\Pi|
\end{aligned}
$$

We now use these inequalities to find a lower bound for $\mu^{*}\left(\Pi^{O P T}\right)$. Picking up where we left off: 


$$
\begin{aligned}
\mu^{*}\left(\Pi^{O P T}\right) & =\sum_{\pi \in \Pi^{O P T}} \mu^{*}(\pi) \\
& =\sum_{b \in \Pi^{O P T}} \mu^{*}(b)\left[\sum_{\pi \in \Pi^{O P T}} A^{\gamma_{1}, \gamma_{2}}(b, \pi)\right]+\sum_{c \in \Pi \backslash \Pi^{O P T}} \mu^{*}(c)\left[\sum_{\pi \in \Pi^{O P T}} A^{\gamma_{1}, \gamma_{2}}(c, \pi)\right] \\
& \geq \sum_{b \in \Pi^{O P T}} \mu^{*}(b)\left[\frac{\left|\Pi^{O P T}\right|}{|\Pi|} \gamma_{1}^{N}+\left(1-\gamma_{1}\right)^{N}\right]+\sum_{c \in \Pi \backslash \Pi^{O P T}} \mu^{*}(c)\left[\left(\gamma_{1}+\gamma_{2}\right)^{N} \frac{\left|\Pi^{O P T}\right|}{|\Pi|}\right] \\
& =\left[\frac{\left|\Pi^{O P T}\right|}{|\Pi|} \gamma_{1}^{N}+\left(1-\gamma_{1}\right)^{N}\right] \mu^{*}\left(\Pi^{O P T}\right)+\left[\left(\gamma_{1}+\gamma_{2}\right)^{N} \frac{\left|\Pi^{O P T}\right|}{|\Pi|}\right]\left(1-\mu^{*}\left(\Pi^{O P T}\right)\right)
\end{aligned}
$$

Rearranging terms, this implies that

$$
\begin{aligned}
& \mu^{*}\left(\Pi^{O P T}\right)\left(1-\left[\frac{\left|\Pi^{O P T}\right|}{|\Pi|} \gamma_{1}^{N}+\left(1-\gamma_{1}\right)^{N}\right]+\left[\left(\gamma_{1}+\gamma_{2}\right)^{N} \frac{\left|\Pi^{O P T}\right|}{|\Pi|}\right]\right) \geq\left[\left(\gamma_{1}+\gamma_{2}\right)^{N} \frac{\left|\Pi^{O P T}\right|}{|\Pi|}\right] \\
& \mu^{*}\left(\Pi^{O P T}\right) \geq \frac{\left(\left[\left(\gamma_{1}+\gamma_{2}\right)^{N} \frac{\left|\Pi^{O P T}\right|}{|\Pi|}\right]\right)}{\left(1-\left[\frac{\left|\Pi^{O P T}\right|}{|\Pi|} \gamma_{1}^{N}+\left(1-\gamma_{1}\right)^{N}\right]+\left[\left(\gamma_{1}+\gamma_{2}\right)^{N} \frac{\left|\Pi \Pi^{O P T}\right|}{|\Pi|}\right]\right)}=: f\left(\gamma_{1}, \gamma_{2}\right)
\end{aligned}
$$

The numerator and the denominator are continuous functions in $\gamma_{1}$ and $\gamma_{2}$, and provided $\gamma_{2}>0$, the denominator is non-zero at $\gamma_{1}=0$. Taking the limit as $\gamma_{1} \rightarrow 0$, continuity gives us the following lemma:

\section{Lemma 3.4.1:}

For any $\epsilon>0$, there exists $\gamma_{\epsilon} \in(0,1)$ such that $\gamma_{1} \in\left(0, \gamma_{\epsilon}\right)$ yields

$$
\mu_{\gamma_{1}, \gamma_{2}, \lambda}^{*}\left(\Pi^{O P T}\right) \geq 1-\epsilon
$$

Proof:

$$
\lim _{\gamma_{1} \rightarrow 0} f\left(\gamma_{1}, \gamma_{2}\right)=\frac{\gamma_{2}^{N} \cdot \frac{\left|\Pi^{O P T}\right|}{|\Pi|}}{\gamma_{2}^{N} \cdot \frac{\left|\Pi^{O P T}\right|}{|\Pi|}}=1
$$

Hence for $\epsilon>0$, there exists $\delta>0:\left|\gamma_{1}-0\right|<\delta \Rightarrow f\left(\gamma_{1}, \gamma_{2}\right) \geq 1-\epsilon$. Since 
$\mu_{\gamma_{1}, \gamma_{2}}^{*} \geq f\left(\gamma_{1}, \gamma_{2}\right)$, taking $\gamma_{1}<\delta=: \gamma_{\epsilon}$ completes the proof of lemma 3.4.1.

\subsection{Results on Similar Matrices}

Consider $J$, the transition matrix for an irreducible, aperiodic Markov chain on a finite set of states, $X$, with $|X|=r$. Then, there exists a unique probability distribution $\mu^{*}$ on $X$ such that $\mu^{*}=\mu^{*} J$, and moreover it will be shown that for any initial distribution $\mu_{0}$,

$$
\lim _{n \rightarrow \infty} \mu_{0} J^{n}=\mu^{*}
$$

Now suppose that we are given a sequence $\left\{H_{n}\right\}_{n \geq 0}$ of transition kernels that satisfies $\left|H_{n}(i, j)-J(i, j)\right|<\epsilon$ for every $i, j \in X$ and every $n \in \mathbb{N}$, and wish to know about the behaviour of the trajectory $\left\{\mu_{k}\right\}_{k \geq 0}$, where the points in the sequence are given by

$$
\mu_{k+1}=\mu_{0} H_{0} H_{1} \cdots H_{k}
$$

The intuition is such: if we can control how similar $H_{n}$ 's are to $J$, then the trajectory $\mu_{k}$ will tend to a small neighbourhood containing $\mu^{*}$. This section formalizes this intuition.

\section{Definitions and Previous Results}

Definition: Let $P, Q$ be $r \times r$ matrices, and let $\tau>0$ be given. We say that $P$ is $\tau$-similar to $Q$ if $|P(i, j)-Q(i, j)| \leq \tau$ for every row $i$, and every column $j$.

Definition: The Dobrushin coefficient for a transition kernel $P$ is denoted $\delta(P)$ and 
is given by

$$
\delta(P):=\min _{i, k} \sum_{j \in X} \min \{P(i, j), P(k, j)\}
$$

The proof of the following fact can be found on p. 25-26 of [17].

Fact 3.5.1: Let $P$ be a transition kernel on the set of states $X$, and let $\mu, \nu$ be probability measures on $X$, we have

$$
\|\mu P-\nu P\|_{1} \leq(1-\delta(P))\|\mu-\nu\|_{1}
$$

For any transition kernel $P$, we have $\delta(P) \in[0,1]$. So, if $\delta(P)>0$, then $P$ is a contraction and has a unique fixed point $\mu^{*}=\mu^{*} P$. We leverage this to prove the well-known result $\lim _{n} \mu_{0} P^{n}=\mu^{*}$ :

$$
\begin{gathered}
\left\|\mu_{k+1}-\mu^{*}\right\|_{1}=\left\|\mu_{k} P-\mu^{*} P\right\|_{1} \leq(1-\delta(P))\left\|\mu_{k}-\mu^{*}\right\|_{1} \\
\Rightarrow\left\|\mu_{k}-\mu^{*}\right\|_{1} \leq(1-\delta(P))^{k}\left\|\mu_{0}-\mu^{*}\right\|_{1}
\end{gathered}
$$

Thus, $\lim _{k \rightarrow \infty}\left\|\mu_{k}-\mu^{*}\right\|_{1}=0 \Rightarrow \lim _{k \rightarrow \infty} \mu_{k}=\mu^{*}$.

Lemma 3.5.2: If $Q$ is $\epsilon$-similar to $P$, then $|\delta(P)-\delta(Q)| \leq r \epsilon$, where $r=|X|$.

Proof: for any $i, j, k \in X$, we have

$$
\min \{Q(i, j), Q(k, j)\} \geq \min \{P(i, j)-\epsilon, P(k, j)-\epsilon\}=\min \{P(i, j), P(k, j)\}-\epsilon
$$


Summing over $j \in X$, we have

$$
\begin{gathered}
\sum_{j} \min \left\{Q(i, j), Q(k, j) \geq\left(\sum_{j} \min \{P(i, j), P(k, j)\}\right)-r \epsilon\right. \\
\Rightarrow \delta(Q):=\min _{i, k} \sum_{j} \min \left\{Q(i, j), Q(k, j) \geq \min _{i, k}\left(\sum_{j} \min \{P(i, j), P(k, j)\}\right)-r \epsilon=\delta(P)-r \epsilon\right.
\end{gathered}
$$

Symmetrically, $\delta(P) \geq \delta(Q)-r \epsilon$, completing the proof of lemma 3.5.2. 
The next result is key to the proof of the main theorem in section 5 .

\section{Proposition 3.5.3:}

Let $r \in \mathbb{N}$ and let $J$ be an $r \times r$ transition matrix, with states labelled $1,2, \ldots, r$, and with $\delta(J)>0$. (Thus, there exists a unique $\mu^{*}$ satisfying $\mu^{*}=\mu^{*} J$ ).

Let $\Delta>0$ be given, and let $\left\{H_{n}\right\}_{n \geq 0}$ be a sequence of matrices that are $\epsilon$-similar to $J$, with

$$
\epsilon<\min \left\{\frac{\delta(J)}{2 r}, \frac{\delta(J) \Delta}{4 r}\right\}
$$

Consider the sequence $\left\{\mu_{k}\right\}_{k \geq 0}$ given by

$$
\begin{aligned}
\mu_{0} & \text { an arbitrary probability distribution on the set }\{1,2, \ldots, r\} \\
\mu_{k+1}=\mu_{k} H_{k} & \text { for all } k \geq 1
\end{aligned}
$$

Then, we have

$$
\limsup _{n \rightarrow \infty}\left\|\mu_{n}-\mu^{*}\right\|_{1}<\Delta
$$




\section{Proof of Proposition 3.5.3}

By Lemma 3.5.2, we have $\left|\delta(J)-\delta\left(H_{k}\right)\right| \leq r \epsilon$. This together with our choice $\epsilon<\frac{\delta(J)}{2 r}$ implies

$$
\sup \left\{1-\delta\left(H_{k}\right): k \geq 0\right\} \leq 1-\frac{\delta(J)}{2}=: \rho<1
$$

Now, let us denote $H_{n}=J-E_{n}$, where the matrix of error terms $E_{n}$ is $\epsilon$-similar to the all-zeros matrix. We have that

$$
\begin{aligned}
\left\|\mu_{1}-\mu^{*}\right\|_{1} & =\left\|\mu_{0} H_{0}-\mu^{*} J\right\|_{1} \\
& \left.=\left\|\mu_{0} H_{0}-\mu^{*} H_{0}+\mu^{*} E_{0}\right\|_{1} \quad \text { (using } J=H_{n}-E_{n}\right) \\
& \leq\left\|\mu_{0} H_{0}-\mu^{*} H_{0}\right\|_{1}+\left\|\mu^{*} E_{0}\right\|_{1} \quad \text { (triangle inequality) } \\
& \left.\leq\left\|\mu_{0}-\mu^{*}\right\|_{1}\left(1-\delta\left(H_{0}\right)\right)+\left\|\mu^{*} E_{0}\right\|_{1} \quad \text { (using Fact } 3.5 .1\right) \\
& \leq\left\|\mu_{0}-\mu^{*}\right\|_{1}\left(1-\delta\left(H_{0}\right)\right)+r \epsilon \quad \text { (since } \mu^{*} \text { is a probability vector) } \\
& \leq\left\|\mu_{0}-\mu^{*}\right\|_{1} \rho+r \epsilon
\end{aligned}
$$

By induction, we have that

$$
\left\|\mu_{n}-\mu^{*}\right\|_{1} \leq \rho^{n}\left\|\mu_{0}-\mu^{*}\right\|_{1}+r \epsilon\left(\sum_{j=0}^{n-1} \rho^{j}\right)
$$

(For completeness, here is the actual induction: Assume the following holds:

$$
\left\|\mu_{n}-\mu^{*}\right\|_{1} \leq \rho^{n}\left\|\mu_{0}-\mu^{*}\right\|_{1}+r \epsilon\left(\sum_{j=0}^{n-1} \rho^{j}\right) .
$$


We will show that this implies

$$
\left\|\mu_{n+1}-\mu^{*}\right\|_{1} \leq \rho^{n+1}\left\|\mu_{0}-\mu^{*}\right\|_{1}+r \epsilon\left(\sum_{j=0}^{(n+1)-1} \rho^{j}\right)
$$

We have that $\left\|\mu_{n+1}-\mu^{*}\right\|_{1}=\left\|\mu_{n} H_{n}-\mu^{*} H_{n}+\mu^{*} E_{n}\right\|_{1} \leq \rho\left\|\mu_{n}-\mu^{*}\right\|_{1}+r \epsilon$. Inserting the inequality in the induction hypothesis completes the induction. So, inequality (3.1) holds.)

The first term on the righthand side of inequality (3.1) decreases monotonically to zero, and in the limit the second term becomes a geometric series with common ratio $\rho$. Our choice of $\epsilon$ then makes this series small:

$$
r \epsilon \sum_{j \geq 0} \rho^{j}=\frac{r \epsilon}{1-\rho}=\frac{r \epsilon}{1-(1-\delta(P) / 2)}=\frac{2 r \epsilon}{\delta(P)}<\frac{\Delta}{2}
$$

The terms in this series are all non-negative, so this is enough to show that the summed error will always be less than $\frac{\Delta}{2}$. Then, since $\rho^{n}\left\|\mu_{0}-\mu^{*}\right\|_{1} \rightarrow 0$ as $n \rightarrow \infty$, there exists $N \in \mathbb{N}$ such that $\rho^{n}\left\|\mu_{0}-\mu^{*}\right\|_{1}<\frac{\Delta}{2}$ for every $n \geq N$.

Together, this implies that $\left\|\mu_{n}-\mu^{*}\right\|_{1}<\Delta$ for every $n \geq N$, hence the result follows

$$
\limsup _{n \rightarrow \infty}\left\|\mu_{n}-\mu^{*}\right\|_{1}<\Delta
$$




\subsection{Parameter Restriction and Supporting Results}

\section{Vector Notation}

Let $\pi_{k}$ denote the baseline joint policy for exploration phase $k$, and let $\Pi^{O P T}$ be the set of all team optimal deterministic joint policies.

Our goal is to make $\liminf _{k \rightarrow \infty} \operatorname{Prob}\left(\pi_{k} \in \Pi^{O P T}\right)$ arbitrarily close to 1 . Let us now introduce some notation:

$$
\begin{aligned}
& \mu_{k}(\pi):=\operatorname{Prob}\left(\pi_{k}=\pi\right) \\
& C_{k}(\pi, \tilde{\pi}):=\operatorname{Prob}\left(\pi_{k+1}=\tilde{\pi} \mid \pi_{k}=\pi\right)
\end{aligned}
$$

Using this notation, we have that $\operatorname{Prob}\left(\pi_{k} \in \Pi^{O P T}\right)=\sum_{\pi \in \Pi \text { OPT }} \mu_{k}(\pi)$. By the law of total probability, we also have

$$
\mu_{k+1}=\mu_{k} C_{k}
$$

The present section will prove that appropriate parameter restrictions make the entries of $C_{k}$ arbitrarily close to the entries of $A_{\lambda}^{\gamma_{1}, \gamma_{2}}$ from section 3.4. (For all but finitely many indices $k$. This will be sufficient for the proof of the main result.) 


\subsection{PARAMETER RESTRICTION AND SUPPORTING RESULTS 46}

\section{Necessary Lemmas and Objects from [1]}

The following lemma can be found in appendix B in [1]. It relates fixed points of (standard) Q-learning in two different MDP settings.

The notation $Q_{\pi^{-i}}^{i}$ here is the fixed point of the Q-factor update $F$ discussed in the background section, and the MDP at hand is the one obtained from the stochastic game where all players except $i$ play the stationary strategy $\pi^{-i}$.

We borrow the notation from [1], where the individual policy $\pi_{k}^{i}$ is the (deterministic, stationary) baseline policy for player $i$ in exploration phase $k$, and the policy $\bar{\pi}_{k}^{i}$ is the (stationary but not deterministic) joint policy that is actually played during an exploration phase-i.e. $\bar{\pi}_{k}^{i}$ is the mixed policy that follows $\pi_{k}^{i}$ with probability $1-\rho^{i}$ and follows a policy of uniformly random action selection with probability $\rho^{i}$.

Lemma 3 in [1]:

Suppose assumption 1 on 25 holds.

For any $\epsilon>0$, there exists $\rho_{\epsilon}>0$ such that $\rho^{i} \in\left(0, \rho_{\epsilon}\right]$ for every player $i$ implies

$$
\left\|Q_{\bar{\pi}_{k}^{-i}}^{i}-Q_{\pi_{k}^{-i}}^{i}\right\|_{\infty} \leq \epsilon \quad \text { for all } i, k
$$

The parameter restrictions we make here also make reference to $\bar{\delta}$ from [1]:

$$
\bar{\delta}:=\min _{i, \pi^{-i}, x}\left\{\left|Q_{\pi^{-i}}^{i}(x, u)-Q_{\pi^{-i}}^{i}(x, v)\right|: u, v \in \mathbb{U}^{i}, \text { and } Q_{\pi^{-i}}^{i}(x, u) \neq Q_{\pi^{-i}}^{i}(x, v)\right\}
$$

This value is the minimum gap between two distinct Q-factors associated to the same state. It will be used in the construction of best response sets, and must be sufficiently small to avoid conflating a non-best-response with a valid best-response. 


\section{Parameter Restrictions}

For intuition's sake, let us overload the notation and use OPT to refer to the optimal score, and refer to the 'next best' score by $N E X T$ :

$$
\begin{aligned}
O P T & :=\sum_{x \in \mathbb{X}} J_{x}\left(\pi^{*}\right)=\sum_{x \in \mathbb{X}} Q_{\pi^{*-i}}^{i}\left(x, \pi^{* i}(x)\right) \\
N E X T & :=\min _{i, \pi \in \Pi \backslash \Pi^{O P T}}\left\{\sum_{x \in \mathbb{X}} Q_{\pi^{-i}}^{i}\left(x, \pi^{i}(x)\right)\right\}
\end{aligned}
$$

$O P T$ is the true score of any optimal policy $\pi^{*}$, and is the lowest attainable score, for any player. (The second equality in the line introducing $O P T$ will be justified below.) $N E X T$ is the second lowest possible score, for any player.

Claim: NEXT > OPT.

First, we argue that if $\pi^{*} \in \Pi^{O P T}$ and $\pi \in \Pi$, then for every $i$

$$
Q_{\pi^{*-i}}^{i}\left(x, \pi^{* i}(x)\right) \leq Q_{\pi^{-i}}^{i}(x, u) \text { for any } u^{i} \in \mathbb{U}^{i}
$$

To see this, we use our background section heavily: by claim 2.10 , if $\pi^{-i}$ is fixed, then $i$ faces a Markov decision process that has an optimal policy $\pi_{B R}^{i}$. Denote the fixed point of the Q-learning process by $Q_{\pi^{-i}}^{i}$, and denote player $i$ 's value function for this MDP by $V_{\pi^{-i}}^{*}$. By Lemma 2.7, we have

$$
V_{\pi^{-i}}^{*}(x)=\min _{u^{i} \in \mathbb{U}^{i}} Q_{\pi^{-i}}^{i}\left(x, u^{i}\right)
$$

And by Lemmas 2.4-2.6, we have that $J_{x}\left(\left(\pi_{B R}^{i}, \pi^{-i}\right)\right)=V_{\pi^{-i}}^{*}(x)$ for every $x$, since $\pi_{B R}^{i}$ 


\subsection{PARAMETER RESTRICTION AND SUPPORTING RESULTS 48}

is an optimal policy for the MDP.

Next we consider the centralized MDP, in which a single controller interacts with the environment but has action set $\mathbb{U}^{1} \times \cdots \mathbb{U}^{N}$. For this centralized MDP, the policy $\tilde{\pi}=\left(\pi_{B R}^{i}, \pi^{-i}\right)$ is admissible, so $J_{x}(\tilde{\pi}) \geq \inf _{\pi \in \Pi} J_{x}(\pi)=V^{*}(x)$.

We now apply the same reasoning to the optimal policy $\pi^{*}$, and we get that for every $i$

$$
J_{x}\left(\pi^{*}\right)=V^{*}(x)=V_{\pi^{*-i}}^{*}(x)=\min _{u^{i} \in \mathbb{U}^{i}} Q_{\pi^{*}-i}^{i}\left(x, u^{i}\right)
$$

(This also shows the equality from the top: $\sum_{x \in \mathbb{X}} J_{x}\left(\pi^{*}\right)=\sum_{x \in \mathbb{X}} Q_{\pi^{*-i}}^{i}\left(x, \pi^{* i}(x)\right)$.) This proves the following fact about Q-factors: if $\pi^{*}$ is a team optimal policy and $\pi \in \Pi$, then for every player $i$

$$
Q_{\pi^{*-i}}^{i}\left(x, \pi^{* i}(x)\right) \leq Q_{\pi^{-i}}^{i}(x, u) \text { for any } u^{i} \in \mathbb{U}^{i}
$$

This implies that $N E X T \geq O P T$. Suppose $N E X T=O P T$, and the minimizing policy was $\pi \in \Pi \backslash \Pi^{O P T}$. Then $J_{x}(\pi)=V^{*}(x)=\inf _{\gamma \in \Delta} J_{x}(\pi)$ for every state $x$, and by definition $\pi \in \Pi^{O P T}$, a contradiction. So indeed, $N E X T>O P T$.

\section{First parameter restriction}

On the tolerance for sub-optimality, $d$ :

$$
d:=\frac{(N E X T-O P T)}{4}
$$

$d$ is used by the player when deciding whether a baseline joint policy is optimal or not. If a policy's score is near the optimal score, $d$ gives us a small cushion to account for 


\subsection{PARAMETER RESTRICTION AND SUPPORTING RESULTS 49}

noise (since Q-learning is stopped after a finite number of steps, and convergence to the fixed point will not obtain exactly), but not so large a cushion as to misinterpret suboptimal policies as optimal.

The learning rule uses a second parameter for tolerance for sub-optimality, $\delta^{i}$. This parameter is used only for constructing best response sets at the end of an exploration phase. It too needs to be positive but not too big, for the same reason as $d$. We enforce

Second parameter restriction

On the tolerance for sub-optimality, $\delta^{i}$ :

$$
\delta^{i} \in(0, \bar{\delta}) \quad \text { for every player } i
$$

The next parameter restriction concerns the experimentation probabilities, $\rho^{i}$. As noted in the literature review, multi-agent learning is difficult because players at once must learn and teach (see [10]). For Q-learning to work, we needed a stationary environment. In decentralized Q-learning, each player faces a stationary environment at time $k$, given by $\bar{\pi}_{k}^{-i}$, and this stationary environment approximates the stationary environment induced by players following $\pi_{k}^{-i}$. The experimentation probabilities must be kept sufficiently small, in order for this approximation to be good. That is, players must reduce their own experimentation probabilites in order to teach the others about the (baseline) environment.

We use Lemma 3 in $\left[1\right.$, Appendix B] to assert the existence of $\bar{\rho}_{1}$ and $\bar{\rho}_{2}$ below: 
We will use this as follows: define

$$
\begin{array}{r}
\bar{\rho}_{1}: \rho^{i} \leq \bar{\rho}_{1} \forall i \Rightarrow\left\|Q_{\bar{\pi}_{k}^{-i}}^{i}-Q_{\pi_{k}^{-i}}^{i}\right\|_{\infty}<\frac{1}{4} \min _{i}\left\{\delta^{i}, \bar{\delta}-\delta^{i}\right\} \text { for every } i, k \\
\bar{\rho}_{2}: \rho^{i} \leq \bar{\rho}_{2} \forall i \Rightarrow\left\|Q_{\bar{\pi}_{k}^{-i}}^{i}-Q_{\pi_{k}^{-i}}^{i}\right\|_{\infty}<\frac{d}{4|\mathbb{X}|} \text { for every } i, k
\end{array}
$$

(Note: these inequalities are now strict. This is intentional, and does not pose a problem, since we could take $\epsilon^{\prime}=\frac{\epsilon}{2}$ when invoking the lemma and get a strict inequality with $\epsilon$ on the right side. Lemma 3 still asserts that such $\bar{\rho}_{1}$ and $\bar{\rho}_{2}$ exist.) Now define $\bar{\rho}:=\min \left\{\bar{\rho}_{1}, \bar{\rho}_{2}\right\}$

Third parameter restriction On the experimentation probabilities:

$$
\rho^{i} \in(0, \bar{\rho}) \quad \text { for every } i
$$

\section{Comparing actual Transition Probabilities with $A_{\lambda}^{\gamma_{1}, \gamma_{2}}$}

With these parameter restrictions in place, we turn our attention back to the proof of the theorem. We wish to select parameters in such a way as to make the conditional probability matrices $C_{k}$ similar to $A_{\lambda}^{\gamma_{1}, \gamma_{2}}$.

Recall that $A_{\lambda}^{\gamma_{1}, \gamma_{2}}\left(\pi, \pi^{\prime}\right)$ was defined to be the probability of this transition using an oracle: the oracle first (correctly) tells players whether or not $\pi$ is an optimal policy, and then the oracle gives players their (correct) best response sets. From there, the player chooses their next baseline policy as specified in section 3.4. 


\subsection{PARAMETER RESTRICTION AND SUPPORTING RESULTS 51}

Our strategy going forward is such: first, we define an event that can replace an oracle.

The event will occur when (i) every player $i$ will have a cutoff estimate $\Lambda_{B}^{i}$ that is close enough to the optimal score, $O P T$. In particular, we will ask for $\frac{d}{2}$ closeness; (ii) for every player, the observed score (a random variable) and the true score of that baseline joint policy are close enough so the cutoff appropriately classifies the baseline as optimal or not. In particular, we will require again that they are within $\frac{d}{2}$; finally, (iii) the observed Q-factors are close enough to the true values that the recovered best response set coincides exactly with the true best response set.

In this event, the two requirements (i) and (ii), replace the oracle's ability to classify the joint baseline policy as optimal or not correctly. Point (iii) replaces the oracle's ability to provide the true best response set.

The second step will be to show that this event described above occurs with sufficiently high probability. This will allow us to achieve the necessary similarity, and invoke proposition 3.5.3.

\section{Relevant Events}

First we define an event $Y_{k}$ relevant to cutoff information:

$$
Y_{k}:=\left\{\omega \in \Omega: \Lambda_{B}^{i}(\omega) \in\left(O P T-\frac{d}{2}, O P T+\frac{d}{2}\right) \text { for every player } i\right\}
$$

Where $k$ is in the $(B+1)^{t h}$ block, i.e. $k \in\{(B+1) \cdot W,(B+1) \cdot W+1, \ldots,(B+1)$. $W+W-1\}$, and hence players use $\Lambda_{B}^{i}$ to do their updating after exploration phase $k$. 


\subsection{PARAMETER RESTRICTION AND SUPPORTING RESULTS 52}

The event $Y_{k}$ implies that all players have accurate cutoff information throughout the $(B+1)^{t h}$ block.

Next, we define two events, $E_{k}$ and $F_{k}$, related to Q-factors:

$$
\begin{aligned}
& E_{k}:=\left\{\omega \in \Omega:\left|S_{k}^{i}-\sum_{x \in \mathbb{X}} Q_{\pi_{k}^{-i}}^{i}\left(x, \pi_{k}^{i}(x)\right)\right|<\frac{d}{2} \quad \text { for every } i=1, \ldots, N\right\} \\
& F_{k}:=\left\{\omega \in \Omega:\left\|Q_{t_{k+1}}^{i}-Q_{\pi_{k}^{i}}^{i}\right\|_{\infty}<\frac{1}{2} \min _{i=1, \ldots, N}\left\{\delta^{i}, \bar{\delta}-\delta^{i}\right\}\right\}
\end{aligned}
$$

The event $F_{k}$ together with this parameter restriction implies that a state-action pair will be included in the (obtained) best response set if and only if it belongs to the real best response set.

The event $R_{k}:=Y_{k} \cap E_{k} \cap F_{k}$ gives us the following: (i) all players are using appropriate cutoffs; (ii) using this cutoff and $E_{k}$, all players correctly assess whether or not the current baseline policy is optimal; (iii) all players have correct best response sets. I.e. their obtained best response set is exactly equal to the set of true best responses.

Our restriction on $d$ was made so that $O P T+\frac{d}{2}+d<N E X T-\frac{d}{2}$. (In the event that the cutoff is in the interval $\left(O P T-\frac{d}{2}, O P T+\frac{d}{2}\right)$ and the score is within $\frac{d}{2}$ of its true value, i.e. $\left|S_{k}^{i}-\sum_{x} Q_{t_{k+1}}^{i}\left(x, \pi_{k}^{i}(x)\right)\right|<\frac{d}{2}$, this restriction on $d$ is enough to distinguish between optimal and suboptimal policies.) I.e., we need $d<(N E X T-O P T) / 2$. With these points explained, it is clear that

$$
A_{\lambda}^{\gamma_{1}, \gamma_{2}}\left(\pi, \pi^{\prime}\right)=\operatorname{Prob}\left(\pi_{k+1}=\pi^{\prime} \mid \pi_{k}=\pi, R_{k}\right)
$$

where $A_{\lambda}^{\gamma_{1}, \gamma_{2}}\left(\pi, \pi^{\prime}\right)$ is not the unconditional probability of transitioning, but rather the transition kernel of the idealized Markov chain presented in section 3.4. 


\subsection{PARAMETER RESTRICTION AND SUPPORTING RESULTS 53}

\section{Lemma 3.6.1: A sufficient condition to obtain similar matrices}

Let $Y_{k}, E_{k}, F_{k}$, be the sets defined on pages 51 and 52 .

Define $R_{k}=Y_{k} \cap E_{k} \cap F_{k}$.

If $\operatorname{Prob}\left(R_{k} \mid \pi_{k}=\pi\right) \geq 1-\tau$ for every $\pi \in \Pi$, then $C_{k}$ is $\tau$-similar to $A_{\lambda}^{\gamma_{1}, \gamma_{2}}$.

Proof:

Let $R_{k}^{C}$ be the complement of $R_{k}$. By the law of total probability,

$$
\begin{gathered}
\operatorname{Prob}\left(\pi_{k+1}=\pi^{\prime} \mid \pi_{k}=\pi\right)=\operatorname{Prob}\left(\pi_{k+1}=\pi^{\prime} \mid \pi_{k}=\pi, R_{k}\right) \operatorname{Prob}\left(R_{k} \mid \pi_{k}=\pi\right)+ \\
\operatorname{Prob}\left(\pi_{k+1}=\pi^{\prime} \mid \pi_{k}=\pi, R_{k}^{C}\right) \operatorname{Prob}\left(R_{k}^{C} \mid \pi_{k}=\pi\right)
\end{gathered}
$$

Since $\operatorname{Prob}\left(R_{k} \mid \pi_{k}=\pi\right) \geq 1-\tau$, we have

$$
\begin{aligned}
\operatorname{Prob}\left(\pi_{k+1}=\pi^{\prime} \mid \pi_{k}=\pi\right) & \geq \operatorname{Prob}\left(\pi_{k+1}=\pi^{\prime} \mid \pi_{k}=\pi, R_{k}\right) \operatorname{Prob}\left(R_{k} \mid \pi_{k}=\pi\right)+0 \\
& \geq \operatorname{Prob}\left(\pi_{k+1}=\pi^{\prime} \mid \pi_{k}=\pi, R_{k}\right)[1-\tau] \\
& =\operatorname{Prob}\left(\pi_{k+1}=\pi^{\prime} \mid \pi_{k}=\pi, R_{k}\right)-\tau\left(\operatorname{Prob}\left(\pi_{k+1}=\pi^{\prime} \mid \pi_{k}=\pi, R_{k}\right)\right) \\
& \geq \operatorname{Prob}\left(\pi_{k+1}=\pi^{\prime} \mid \pi_{k}=\pi, R_{k}\right)-\tau(1)
\end{aligned}
$$

and

$$
\begin{aligned}
\operatorname{Prob}\left(\pi_{k+1}=\pi^{\prime} \mid \pi_{k}=\pi\right) & \leq \operatorname{Prob}\left(\pi_{k+1}=\pi^{\prime} \mid \pi_{k}=\pi, R_{k}\right) \operatorname{Prob}\left(R_{k} \mid \pi_{k}=\pi\right)+\operatorname{Prob}\left(R_{k}^{C} \mid \pi_{k}=\pi\right) \\
& \leq \operatorname{Prob}\left(\pi_{k+1}=\pi^{\prime} \mid \pi_{k}=\pi, R_{k}\right) \cdot 1+(1-(1-\tau)) \\
& =\operatorname{Prob}\left(\pi_{k+1}=\pi^{\prime} \mid \pi_{k}=\pi, R_{k}\right) \cdot 1+\tau
\end{aligned}
$$

All together, we have $\left|\operatorname{Prob}\left(\pi_{k+1}=\pi^{\prime} \mid \pi_{k}=\pi\right)-\operatorname{Prob}\left(\pi_{k+1}=\pi^{\prime} \mid \pi_{k}=\pi, R_{k}\right)\right| \leq \tau$, and since $C_{k}\left(\pi, \pi^{\prime}\right)=\operatorname{Prob}\left(\pi_{k+1}=\pi^{\prime} \mid \pi_{k}=\pi\right)$ and $\operatorname{Prob}\left(\pi_{k+1}=\pi^{\prime} \mid \pi_{k}=\pi, R_{k}\right)=$ $A_{\lambda}^{\gamma_{1}, \gamma_{2}}\left(\pi, \pi^{\prime}\right)$, the result is obtained. 
Lemma 3.6.2

For any $\tau>0$, we have

$$
\operatorname{Prob}\left(R_{k}\right) \geq 1-\tau \frac{\gamma_{1}^{N}}{|\Pi|} \Rightarrow \operatorname{Prob}\left(R_{k} \mid \pi_{k}=\pi\right) \geq 1-\tau, \text { for every } \pi \in \Pi
$$

Proof: for any $\pi \in \Pi$, we can lower bound the probability $\operatorname{Prob}\left(\pi_{k}=\pi\right) \geq \gamma_{1}^{N} /|\Pi|$ and that way get $\operatorname{Prob}\left(R_{k}\right) \geq 1-\tau \operatorname{Prob}\left(\pi_{k}=\pi\right)$.

First, we argue by contradiction that $\operatorname{Prob}\left(R_{k}\right) \geq 1-\tau \operatorname{Prob}\left(\pi_{k}=\pi\right)$ implies that $\operatorname{Prob}\left(R_{k} \cap\left\{\pi_{k}=\pi\right\}\right) \geq(1-\tau) \operatorname{Prob}\left(\pi_{k}=\pi\right)$ : suppose instead that $\operatorname{Prob}\left(R_{k} \cap\left\{\pi_{k}=\pi\right\}\right)<(1-\tau) \operatorname{Prob}\left(\pi_{k}=\pi\right)$. We have

$$
\begin{aligned}
\operatorname{Prob}\left(R_{k}\right) & =\operatorname{Prob}\left(R_{k} \cap\left\{\pi_{k}=\pi\right\}\right)+\operatorname{Prob}\left(R_{k} \cap\left\{\pi_{k} \neq \pi\right\}\right) \\
& \leq \operatorname{Prob}\left(R_{k} \cap\left\{\pi_{k}=\pi\right\}\right)+\operatorname{Prob}\left(\pi_{k} \neq \pi\right) \quad \text { by set containment } \\
& =\operatorname{Prob}\left(R_{k} \cap\left\{\pi_{k}=\pi\right\}\right)+\left(1-\operatorname{Prob}\left(\pi_{k}=\pi\right)\right) \quad \text { by complementarity } \\
& <(1-\tau) \operatorname{Prob}\left(\pi_{k}=\pi\right)+\left(1-\operatorname{Prob}\left(\pi_{k}=\pi\right)\right) \quad \text { by our supposing not } \\
& =1-\tau \operatorname{Prob}\left(\pi_{k}=\pi\right)
\end{aligned}
$$

$\operatorname{Prob}\left(R_{k}\right)<1-\tau \operatorname{Prob}\left(\pi_{k}=\pi\right)$ contradicts our assumption, by the first line of the proof. So indeed we have $\operatorname{Prob}\left(R_{k} \cap\left\{\pi_{k}=\pi\right\}\right) \geq 1-\tau \operatorname{Prob}\left(\pi_{k}=\pi\right)$. Next our lemma: 


$$
\begin{aligned}
& \operatorname{Prob}\left(R_{k}\right) \geq 1-\tau \frac{\gamma_{1}^{N}}{|\Pi|} \geq 1-\tau \operatorname{Prob}\left(\pi_{k}=\pi\right) \\
\Rightarrow & \operatorname{Prob}\left(R_{k} \cap\left\{\pi_{k}=\pi\right\}\right) \geq(1-\tau) \operatorname{Prob}\left(\pi_{k}=\pi\right) \\
\Rightarrow & \operatorname{Prob}\left(R_{k} \mid \pi_{k}=\pi\right)=\frac{\operatorname{Prob}\left(R_{k} \cap\left\{\pi_{k}=\pi\right\}\right)}{\operatorname{Prob}\left(\pi_{k}=\pi\right)} \geq 1-\tau
\end{aligned}
$$


Lemma 3.6.3: $\operatorname{Prob}\left(R_{k}\right)$ can be made arbitrarily high, by appropriate choice of parameters

Lemma 3.6.3

- Let $\eta>0$ be given

- let $\gamma_{1}, \gamma_{2}>0$ be fixed and given

- let assumption 1 from page 25 hold. (also on p. 62)

- let $d=\frac{N E X T-O P T}{4}$,

- let $\delta^{i} \in(0, \bar{\delta})$ for every $i$,

- let $\rho^{i} \in(0, \bar{\rho}]$ for every $i$.

- let $W \geq \frac{\ln (\eta / 6)}{\ln \left(1-\gamma_{1}^{N} /|\Pi|\right)}$

- let $k$ belong to the $(B+1)^{t h}$ block, $k \in\{B \cdot W, B \cdot W+1, \ldots, B \cdot W+W-1\}$, where $B \geq 1$

There exists $L \in \mathbb{N}$ such that

if $T_{j} \geq L$ for each $j \in\{k,(B-1) \cdot W+0, \ldots,(B-1) \cdot W+W-1\}$, then we have

$\operatorname{Prob}\left(R_{k}\right) \geq 1-\eta$ 


\section{Proof of lemma 3.6.3}

It suffices to show that we can make $\operatorname{Prob}\left(Y_{k}^{C}\right), \operatorname{Prob}\left(E_{k}^{C}\right), \operatorname{Prob}\left(F_{k}^{C}\right)<\frac{\eta}{3}$ :

$$
\begin{aligned}
\operatorname{Prob}\left(R_{k}\right)=\operatorname{Prob}\left(Y_{k} \cap E_{k} \cap F_{k}\right) & =1-\operatorname{Prob}\left(Y_{k}^{C} \cup E_{k}^{C} \cup F_{k}^{C}\right) \\
& \geq 1-\left(\operatorname{Prob}\left(Y_{k}^{C}\right)+\operatorname{Prob}\left(E_{k}^{C}\right)+\operatorname{Prob}\left(F_{k}^{C}\right)\right)
\end{aligned}
$$

For this section, we need to use another lemma from Arslan and Yüksel 2017:

Lemma 2 from [1, appendix B]:

For any $\epsilon>0$ there exists $T_{\epsilon} \in \mathbb{N}$ such that $T_{k} \geq T_{\epsilon}$ implies

$$
P\left(\left\|Q_{t_{k+1}}^{i}-Q_{\bar{\pi}_{k}^{-i}}^{i}\right\|_{\infty} \leq \epsilon \text { for all } i\right) \geq 1-\epsilon
$$

Here, $Q_{t_{k+1}}^{i}$ is the vector of observed Q-factors that the player sees at the end of the $k^{t h}$ exploration phase. On the other hand, $Q_{\bar{\pi}_{k}^{-i}}^{i}$ is the fixed point to the Q-factor update, corresponding to the true MDP that players face (where other players are using mixed, not deterministic, policies). 


\subsection{PARAMETER RESTRICTION AND SUPPORTING RESULTS 58}

We begin by showing $\operatorname{Prob}\left(Y_{k}\right) \geq 1-\frac{\eta}{3}$ :

Let $\mathfrak{B}=\{(B-1) \cdot W+0,(B-1) \cdot W+1, \ldots,(B-1) \cdot W+W-1\}$ be the set of indices of the $B^{\text {th }}$ block, and define

$$
\begin{gathered}
\operatorname{any}_{B}:=\bigcup_{j \in \mathfrak{B}}\left\{\pi_{j} \in \Pi^{O P T}\right\} \\
a l l_{B}:=\bigcap_{j \in \mathfrak{B}}\left\{\left|\left[\sum_{x \in \mathbb{X}} Q_{t_{k+1}}^{i}\left(x, \pi_{j}^{i}(x)\right)\right]-\left[\sum_{x \in \mathbb{X}} Q_{\pi_{j}^{-i}}^{i}\left(x, \pi_{j}^{i}(x)\right)\right]\right|<\frac{d}{2} \text { for every } i\right\}
\end{gathered}
$$

Note that

$$
\operatorname{any}_{B} \cap \operatorname{all}_{B} \subset Y_{k}
$$

The left part of this intersection tells us that at least one optimal policy was visited in the $B^{\text {th }}$ window, and the right part tells us that all of the scores were accurate to within $\frac{d}{2}$. By our choice of $d$ and the definitions of $O P T$ and NEXT, this implies $\Lambda_{B}^{i} \in\left(O P T-\frac{d}{2}, O P T+\frac{d}{2}\right)$ for every $i$.

If we can show that $\operatorname{Prob}\left(a n y_{B}\right) \geq 1-\frac{\eta}{6}$ and $\operatorname{Prob}\left(a l_{B}\right) \geq 1-\frac{\eta}{6}$, then we will have shown that $\operatorname{Prob}\left(Y_{k}\right) \geq 1-\frac{\eta}{3}$.

$$
\operatorname{Prob}\left(a n y_{B}^{C}\right)=\operatorname{Prob}\left(\bigcap_{j \in \mathfrak{B}}\left\{\pi_{j} \notin \Pi^{O P T}\right\}\right)
$$

noting that $\operatorname{Prob}\left(\pi_{j} \in \Pi^{O P T}\right) \geq \frac{\gamma_{1}^{N}}{|\Pi|}$ for any $j$ and that by assumption the random selection process is i.i.d., we compare with sequence of binary random variables, with probability of succeeding $\frac{\gamma_{1}^{N}}{|\Pi|}$, we have 


$$
\operatorname{Prob}\left(\bigcap_{j \in \mathfrak{B}}\left\{\pi_{j} \notin \Pi^{O P T}\right\}\right) \leq\left(1-\frac{\gamma_{1}^{N}}{|\Pi|}\right)^{W} \stackrel{W \rightarrow \infty}{\longrightarrow} 0
$$

Thus, there exists $\tilde{W}: W \geq \tilde{W} \Rightarrow \operatorname{Prob}\left(\bigcap_{j \in \mathfrak{B}}\left\{\pi_{j} \notin \Pi^{O P T}\right\}\right)<\frac{\eta}{6}$. Indeed, this explains our choice of $W$ in the theorem statement: it is simply a rearranging of the inequality $\left.\left(1-\gamma_{1}^{N} /|\Pi|\right)^{W} \leq \eta / 6\right)$ in order to achieve $\operatorname{Prob}\left(a n y_{B}\right) \geq 1-\eta / 6$.

We proceed to $\operatorname{Prob}\left(a l_{B}\right)$. We must invoke Lemma 2 from [1, Appendix B] at this point with $\epsilon$ given by

$$
\epsilon=\min \left\{\frac{d}{4|\mathbb{X}|}, \frac{\eta}{6 W}\right\}
$$

So, there exists $T_{\epsilon} \in \mathbb{N}$ such that $T_{j} \geq T_{\epsilon}$ implies

$$
\operatorname{Prob}\left(\left\|Q_{t_{j+1}}^{i}-Q_{\bar{\pi}_{j}^{-i}}^{i}\right\|_{\infty} \leq \frac{d}{4|\mathbb{X}|} \text { for every } i\right) \geq 1-\epsilon \geq 1-\frac{\eta}{6 W}
$$

Let $\mathcal{T}_{Y}:=T_{\epsilon}$.

By our choice of $\bar{\rho} \leq \bar{\rho}_{2}$, we have $\left\|Q_{\pi_{j}^{-i}}^{i}-Q_{\bar{\pi}_{j}^{-i}}^{i}\right\|_{\infty}<\frac{d}{4|\mathbb{X}|}$ for every $i, j$.

Using the triangle inequality, the event

$$
\left\{\left\|Q_{t_{j+1}}^{i}-Q_{\bar{\pi}_{j}^{-i}}^{i}\right\|_{\infty} \leq \frac{d}{4|\mathbb{X}|} \text { for every i }\right\} \subseteq\left\{\left\|Q_{t_{j+1}}^{i}-Q_{\pi_{j}^{-i}}^{i}\right\|_{\infty}<\frac{d}{2|\mathbb{X}|} \text { for every i }\right\}
$$


Summing over $x \in \mathbb{X}$ gives us the following:

$$
\left\{\left\|Q_{t_{j+1}}^{i}-Q_{\pi_{j}^{-i}}^{i}\right\|_{\infty}<\frac{d}{2|\mathbb{X}|}\right\} \subseteq\left\{\left|\left[\sum_{x \in \mathbb{X}} Q_{t_{j+1}}^{i}\left(x, \pi_{j}^{i}(x)\right)\right]-\left[\sum_{x \in \mathbb{X}} Q_{\pi_{j}^{-i}}^{i}\left(x, \pi_{j}^{i}(x)\right)\right]\right|<\frac{d}{2}\right\}
$$

Now, since

$$
\begin{aligned}
\operatorname{Prob}\left(\operatorname{all}_{B}\right) & =\operatorname{Prob}\left(\bigcap_{j \in \mathfrak{B}}\left\{\left|\left[\sum_{x \in \mathbb{X}} Q_{t_{j+1}}^{i}\left(x, \pi_{j}^{i}(x)\right)\right]-\left[\sum_{x \in \mathbb{X}} Q_{\pi_{j}^{-i}}^{i}\left(x, \pi_{j}^{i}(x)\right)\right]\right|<\frac{d}{2}\right\}\right. \\
& =1-\operatorname{Prob}\left(\bigcup_{j \in \mathfrak{B}}\left\{\left|\left[\sum_{x \in \mathbb{X}} Q_{t_{j+1}}^{i}\left(x, \pi_{j}^{i}(x)\right)\right]-\left[\sum_{x \in \mathbb{X}} Q_{\pi_{j}^{-i}}^{i}\left(x, \pi_{j}^{i}(x)\right)\right]\right| \geq \frac{d}{2}\right\}\right. \\
& \geq 1-\sum_{j \in \mathfrak{B}} \operatorname{Prob}\left(\left\{\left|\left[\sum_{x \in \mathbb{X}} Q_{t_{j+1}}^{i}\left(x, \pi_{j}^{i}(x)\right)\right]-\left[\sum_{x \in \mathbb{X}} Q_{\pi_{j}^{i}}^{i}\left(x, \pi_{j}^{i}(x)\right)\right]\right| \geq \frac{d}{2}\right\}\right) \\
& \geq 1-\sum_{j \in \mathfrak{B}} \frac{\eta}{6 W}=1-\frac{\eta}{6}
\end{aligned}
$$

Thus, $\operatorname{Prob}\left(Y_{k}\right) \geq 1-\frac{\eta}{3}$.

Next, we show $\operatorname{Prob}\left(E_{k}\right) \geq 1-\frac{\eta}{3}$. In fact, this can be done more easily than the previous case. We invoke Lemma 2 from [1] again, this time with (greater) $\epsilon=$ $\min \left\{\frac{d}{4|\mathbb{X}|}, \frac{\eta}{3}\right\}$

By Lemma 2, there exists $T_{\epsilon} \in \mathbb{N}$ such that $T_{k} \geq T_{\epsilon}$ implies

$$
\operatorname{Prob}\left(\left\|Q_{t_{k+1}}^{i}-Q_{\bar{\pi}_{k}^{-i}}^{i}\right\|_{\infty} \leq \frac{d}{4|\mathbb{X}|} \text { for every } i\right) \geq 1-\epsilon \geq 1-\frac{\eta}{3}
$$

Thus, by the same argument as above (choice of $\bar{\rho}_{2}$, triangle inequality, event contaiment), we have 


\subsection{PARAMETER RESTRICTION AND SUPPORTING RESULTS 61}

$\operatorname{Prob}\left(E_{k}\right)=\operatorname{Prob}\left(\left[\sum_{x \in \mathbb{X}} Q_{t_{j+1}}^{i}\left(x, \pi_{j}^{i}(x)\right)\right]-\left[\sum_{x \in \mathbb{X}} Q_{\pi_{j}^{-i}}^{i}\left(x, \pi_{j}^{i}(x)\right)\right] \mid<\frac{d}{2}\right.$ for every $\left.i\right) \geq 1-\frac{\eta}{3}$

Thus, $T_{k} \geq T_{\epsilon}=: \mathcal{T}_{E} \Rightarrow P\left(E_{k}^{C}\right)<\frac{\eta}{3}$, as desired.

Finally, we show that $\operatorname{Prob}\left(F_{k}\right) \geq 1-\frac{\eta}{3}$ :

We apply Lemma 2 now to

$$
\epsilon=\min \left\{\frac{1}{4} \min \left\{\delta^{i}, \bar{\delta}-\delta^{i}: i=1, \ldots, N\right\}, \frac{\eta}{3}\right\}
$$

By our choice of $\bar{\rho} \leq \bar{\rho}_{1}$, we have that

$$
\left\|Q_{\pi_{k}^{-i}}^{i}-Q_{\bar{\pi}_{k}^{-i}}^{i}\right\|_{\infty}<\frac{1}{4} \min \left\{\delta^{i}, \bar{\delta}-\delta^{i}: i=1, \ldots, N\right\} \text { for every } i, k
$$

There exists $T_{\epsilon}=: \mathcal{T}_{F}$ such that $T_{k} \geq \mathcal{T}_{F}$ implies

$$
\operatorname{Prob}\left(\left\|Q_{t_{k+1}}^{i}-Q_{\bar{\pi}_{k}^{-i}}^{i}\right\|_{\infty} \leq \frac{1}{4} \min _{i=1, \ldots, N}\left\{\delta^{i}, \bar{\delta}-\delta^{i}\right\} \text { for every } i\right) \geq 1-\epsilon \geq 1-\frac{\eta}{3}
$$

Then, applying the triangle inequality again, we have

$$
\operatorname{Prob}\left(\left\|Q_{t_{k+1}}^{i}-Q_{\pi_{k}^{-i}}^{i}\right\|_{\infty}<\frac{1}{2} \min _{i=1, \ldots, N}\left\{\delta^{i}, \bar{\delta}-\delta^{i}\right\} \text { for every } i\right) \geq 1-\epsilon \geq 1-\frac{\eta}{3}
$$

This event is contained in $F_{k}$.

Thus, selecting $L \geq \max \left\{\mathcal{T}_{Y}, \mathcal{T}_{E}, \mathcal{T}_{F}\right\}$ and asserting that $T_{j} \geq L$ for each exploration phase $j$ in the $B^{t h}$ block and also $T_{k} \geq L$ implies that $P\left(R_{k}\right) \geq 1-\eta$. 


\subsection{Theorem and Proof}

Consider a finite, discounted stochastic team game, and let $\Pi^{O P T}$ denote the set of team optimal joint policies.

Assumption 1: For any $x, x^{\prime} \in \mathbb{X}$, there exists $H \in \mathbb{N}$ and joint actions $\tilde{u}_{0}, \ldots, \tilde{u}_{H}$ such that

$$
P\left(x_{H+1}=x^{\prime} \mid x_{0}=x, u_{j}=\tilde{u}_{j} \text { for every } j \in\{0,1, \ldots, H\}\right)>0
$$

Consider a stochastic team game in which all players use Algorithm $\mathrm{B}$.

Let $\epsilon>0$. Let assumption 1 hold. There exists $\gamma_{1}^{*}, \gamma_{2}^{*}, W^{*}, L^{*}, \bar{\rho}, \bar{\delta}, d^{*}$ (all depending on $\epsilon$ ) such that if

- $\gamma_{1}=\gamma_{1}^{*}$,

- $\gamma_{2}=\gamma_{2}^{*}$,

- $W=W^{*}$,

- $d=d^{*}$,

- $T_{k} \geq L^{*}$ for every $k$,

- $\rho^{i} \in(0, \bar{\rho})$ for every $i$,

- $\delta^{i} \in(0, \bar{\delta})$ for every $i$,

then there exists $M \in \mathbb{N}$ such that

$$
\operatorname{Prob}\left(\pi_{n} \in \Pi^{O P T}\right) \geq 1-\epsilon \text { for every } n \geq M
$$




\section{Proof of main result}

Fix $\gamma_{2}>0$ and set $\gamma_{2}^{*}=\gamma_{2}$. First, we invoke Lemma 3.4.1: there exists $\gamma_{\frac{\epsilon}{2}}>0$ such that $\gamma_{1}<\gamma_{\frac{\epsilon}{2}}$ implies $\mu_{\gamma_{1}, \gamma_{2}}^{*}\left(\Pi^{O P T}\right) \geq 1-\frac{\epsilon}{2}$.

Fix $\gamma_{1}<\gamma_{\frac{\epsilon}{2}}$, and set $\gamma_{1}^{*}=\gamma_{1}$. We will use the same $\gamma_{1}$ throughout.

Second, we define

$$
\xi=\frac{1}{2} \min \left\{\frac{\delta\left(A_{\lambda}^{\gamma_{1}, \gamma_{2}}\right)}{2|\Pi|}, \frac{\delta\left(A_{\lambda}^{\gamma_{1}, \gamma_{2}}\right) \frac{\epsilon}{4}}{4|\Pi|}\right\}
$$

(We will require $\xi$-similarity of conditional probability matrices $C_{k}$ with $A_{\lambda}^{\gamma_{1}, \gamma_{2}}$, and use $\frac{\epsilon}{4}$ as the $\Delta$ in proposition 3.5.3.)

And invoke lemma 3.6.2 with $\tau=\xi$ : if $\operatorname{Prob}\left(R_{k}\right) \geq 1-\xi \frac{\gamma_{1}^{N}}{|\Pi|}$, then $\operatorname{Prob}\left(R_{k} \mid \pi_{k}=\pi\right) \geq$ $1-\xi$ for every $\pi \in \Pi$.

Next, we choose some $W \geq \ln \left(\frac{\xi}{6}\right) / \ln \left(1-\frac{\gamma_{1}^{N}}{|\Pi|}\right)$, and set $W^{*}=W$. Invoke lemma 3.6.3 with $\xi$ and $W^{*}$ to retrieve the exploration phase length $L$, and set $L^{*}=L$.

This achieves $\operatorname{Prob}\left(R_{k}\right) \geq 1-\xi$ for any $k \geq W$. (We begin at $W$ since we do not have a complete window of $W$ observations until after exploration phase $W-1$.)

Thus, $\operatorname{Prob}\left(R_{k} \mid \pi_{k}=\pi\right) \geq 1-\xi$ for every $\pi \in \Pi$. So, the matrices $\left\{C_{k}\right\}_{k \geq W}$ are $\xi$-similar to $A_{\lambda}^{\gamma_{1}, \gamma_{2}}$, and we invoke proposition 3.5.3, with $\Delta=\frac{\epsilon}{4}$.

We have

$$
\limsup _{n \rightarrow \infty}\left\|\mu_{n}-\mu_{\gamma_{1}, \gamma_{2}, \lambda}^{*}\right\|_{1}<\frac{\epsilon}{4}
$$

Hence, there exists $M \in \mathbb{N}$ such that $n \geq M \Rightarrow\left\|\mu_{n}-\mu_{\gamma_{1}, \gamma_{2}}^{*}\right\|_{1}<\frac{\epsilon}{2}$. 
Then, for $n \geq M$, we have

$$
\begin{aligned}
\left|\mu_{n}\left(\Pi^{O P T}\right)-\mu_{\gamma_{1}, \gamma_{2}}^{*}\left(\Pi^{O P T}\right)\right| & =\left|\sum_{\pi \in \Pi O P T} \mu_{n}(\pi)-\sum_{\pi \in \Pi O P T} \mu_{\gamma_{1}, \gamma_{2}}^{*}(\pi)\right| \\
& \leq \sum_{\pi \in \Pi^{O P T}}\left|\mu_{n}(\pi)-\mu_{\gamma_{1}, \gamma_{2}}^{*}(\pi)\right| \\
& \leq \sum_{\pi \in \Pi}\left|\mu_{n}(\pi)-\mu_{\gamma_{1}, \gamma_{2}}^{*}(\pi)\right|=\left\|\mu_{n}-\mu_{\gamma_{1}, \gamma_{2}}^{*}\right\|_{1} \\
& <\frac{\epsilon}{2}
\end{aligned}
$$

All together, we have $\mu_{n}\left(\Pi^{O P T}\right) \geq 1-\epsilon$, as desired. $\square$ 


\section{Chapter 4}

\section{Simulation Study}

In order to simulate Algorithm B and check whether we could guarantee that the optimal policy would be played with high probability, we devised a small but nontrivial stochastic game, described below.

Using both analysis and experiment, suitable $\bar{\delta}, d>0$, and $W \in \mathbb{N}$ were selected, and the performance of the algorithm was observed for various values of $\gamma_{1}$.

For additional comparison, we wrote another simulation for the oracle process' Markov Chain presented in chapter 3 , and ran it to observe performance of various $\gamma_{1}$ 's.

\section{The Toy Game}

We considered a stochastic game with the following specifications:

- A set of states $\mathbb{X}=\{1,2\}$

- Two players, labelled 1,2.

- A set of control actions $\mathbb{U}^{i}=\{1,2\}$ for each player $i \in\{1,2\}$ 
- A common discount factor $\beta=0.8$

- Stage games for each state $x \in\{1,2\}$, presented below, where players incur costs and seek to minimize long term expected discounted cost.

- A Markov transition kernel, $P\left[\cdot \mid x_{t}, u_{t}^{1}, u_{t}^{2}\right]$ given by the following rule:

$$
\begin{aligned}
& P\left[x_{t+1}=1 \mid u_{t}^{1}=u_{t}^{2}=x_{t}\right]=0.95 \\
& P\left[x_{t+1}=2 \mid u_{t}^{1}=u_{t}^{2}=x_{t}\right]=0.05 \\
& P\left[x_{t+1}=1 \mid \neg\left(u_{t}^{1}=u_{t}^{2}=x_{t}\right)\right]=0.05 \\
& P\left[x_{t+1}=2 \mid \neg\left(u_{t}^{1}=u_{t}^{2}=x_{t}\right)\right]=0.95
\end{aligned}
$$

The transition kernel is constructed to satisfy this requirement: if players are in state 1 , and they coordinate on playing action 1 , then they will return to state 1 with high probability; if players are in state 2, and they coordinate on playing action 2 , then they will leave to state 1 with high probability. Any other behaviour (failure to coordinate, or coordinating on the wrong action) will lead to state 2 with high probability.

\section{The Stage Games:}

\begin{tabular}{|c|c|c|}
\hline $\mathrm{x}=1$ & 1 & 2 \\
\hline 1 & +1 & +3 \\
\hline 2 & +3 & +1 \\
\hline
\end{tabular}

\begin{tabular}{|c|c|c|}
\hline$x=2$ & 1 & 2 \\
\hline 1 & +10 & +10 \\
\hline 2 & +10 & +13 \\
\hline
\end{tabular}

State 1 is a low cost state, in which coordinating one action 1 is optimal; when in state 2 , players can escape state 2 with high probability by coordinating on the costly action 2. This ability to leave state two highlights a tradeoff: incurring a higher cost in this period will likely lead to lower costs in the coming periods. 


\section{The Equilibrium Policies}

Let us denote DM $j$ 's policy "play action $s_{1}$ in state 1 , and play action $s_{2}$ in state 2" with the notation $\left(s_{1}^{j}, s_{2}^{j}\right)$, and then write a joint policy by writing DM1's policy first: $\left[\left(s_{1}^{1}, s_{2}^{1}\right),\left(s_{1}^{2}, s_{2}^{2}\right)\right]$.

So, $[(1,1),(2,1)]$ means that player 1 plays action 1 in every state, and player 2 plays action 2 when in state 1 , and plays action 1 when in state 2 .

Since each player has four deterministic policies, namely $(1,1),(1,2),(2,1)$ and $(2,2)$, there are sixteen deterministic joint policies in total. Of those, four are (deterministic) equilibrium policies: $[(1,1),(1,1)],[(1,2)(1,2)],[(2,1),(2,1)],[(2,2),(2,2)]$.

The game is completely symmetric, so to see why each of these joint policies constitutes an equilibrium, we can fix the policy of the column player and analyze the row player's best responses.

For example, the joint policy $[(1,2)(1,2)]$ is team optimal, given the specified $\beta=0.8$. When the column player commits to playing action 1 in state 1 , the row player would prefer to play 1 in the short term, since it yields the lowest cost. Moreover, playing 1 in response to 1 in state 1 will very likely lead to returning to the low-cost state 1 , and reduces the probability of transitioning to the high-cost state 2 . The analysis of state 2's behaviour is more subtle: if the column player commits to playing action 2 in state 2 , then the row player is faced with a dilemma: playing action 1 and only paying 10 today on the one hand, or playing action 2 and paying 13 on the other. $\beta=0.8$ means that the row player values the future relatively highly, and benefits from paying a small cost immediately in the hope of transitioning to the low-cost state 1 , where significant savings can be had. 
A similar analysis can be applied to the other three equilibrium policies. For illustration, let us also consider one suboptimal equilibrium: $[(2,2),(2,2)]$. Here, the column player will play action 2 no matter the state. The row player's optimal action in state 1 is clear: he should play 2 , since they will transition to the high-cost state with equally high probability no matter what the row player does, so he might as well enjoy a lower cost now. In state 2 , the row player faces the same trade-off as before, and still values the prospect of going to the low-cost state enough to make playing action 2 preferable to action 1 .

The remaining two equilibrium policies can be analyzed using the same method. That they too are equilibrium policies has been checked by computer and by hand.

\section{Parameter Selection}

As noted above, $\bar{\delta}, d, T_{k}$ and $W$ were selected by both analyzing the game and running a few simulations to determine the variance of the Q-factors. Here is a list of parameter selection:

- $\rho^{i}=0.05$ for $i=1,2$

- $\lambda^{i}=0.5$ for $i=1,2$

- $\delta^{i}=1.5$ for $i=1,2$

- $d=10$

- $\gamma_{2}=0.1$

- exploration phase length $T_{k}=10,000$ for every $k$

- window length $W=30$ 
(At this point, an observation on the window length $W$ should mentioned: the restrictions on $W$ in the results of chapter 3 are very loose. In principle, we should have been using $W \approx 70000$, so the comparison to the binary random variable would be useful. However, that approach is overly conservative.)

From there, we considered four values for $\gamma_{1}$ : each of $\gamma_{1} \in\{0.05,0.01,0.005,0.001\}$, with the understanding that the smaller $\gamma_{1}$ was taken to be, the greater the (asymptotic) probability of the baseline policy being optimal.

For the sake of illustrating the method of proof, we also simulated the exact Markov chain from section 3.4, for $\gamma_{2}=0.1$ and the various values of $\gamma_{1}$, to be used for comparison with the Algorithm B's output.

\section{Simulation Specifics}

For a single trial of Algorithm B's performance, play was allowed to run for 480 exploration phases. We constructed a binary variable to record whether the policy during exploration phase $k \in\{1, \ldots, 480\}$ was optimal. To get an idea of $P\left(\pi_{k} \in\right.$ $\Pi^{O P T}$ ), we conducted 500 trials the simulation of algorithm B for a single value of $\gamma_{1}$, and in that way got 500 vectors of size $1 \times 480$. We took the average of each time index, and plotted the relative frequency against the time index

In more detail, each trial $j \in\{1,2, \ldots, 500\}$ began with fixed $\gamma_{1}$ and returned a vector called Ledger ${ }_{j}$ of length 500 whose components were $\{0,1\}$-valued, where $\operatorname{Ledger}_{j}(m)=1$ means that during the $j^{\text {th }}$ trial, the baseline policy $\pi_{m}$ at exploration phase time index $m$ was an optimal policy. 
Then, we used $\left\{\operatorname{Ledger}_{j}\right\}_{j=1}^{500}$ to construct a relative frequency vector with 480 components (one for each exploration phase) called Averages $_{\gamma_{1}}$, where

$$
\operatorname{Averages}_{\gamma_{1}}(m)=\frac{1}{500} \sum_{j=1}^{500} \operatorname{Ledger}_{j}(m)
$$

For comparison, we ran the Markov chain for 480 exploration phases, and repeated this 1000 times, and again considered relative frequency. 


\section{Numerical Results for the Comparison Markov Chain}

Here are the numerical results from the comparison Markov Chain from section 3.4, where players had access to an oracle. The analysis there showed that as $\gamma_{1}$ was taken to 0 , the asymptotic probability of being optimal would increase to 1 . Note that for each of the four values used as $\gamma_{1}$, the probability appears to level off by the $100^{\text {th }}$ exploration phase.

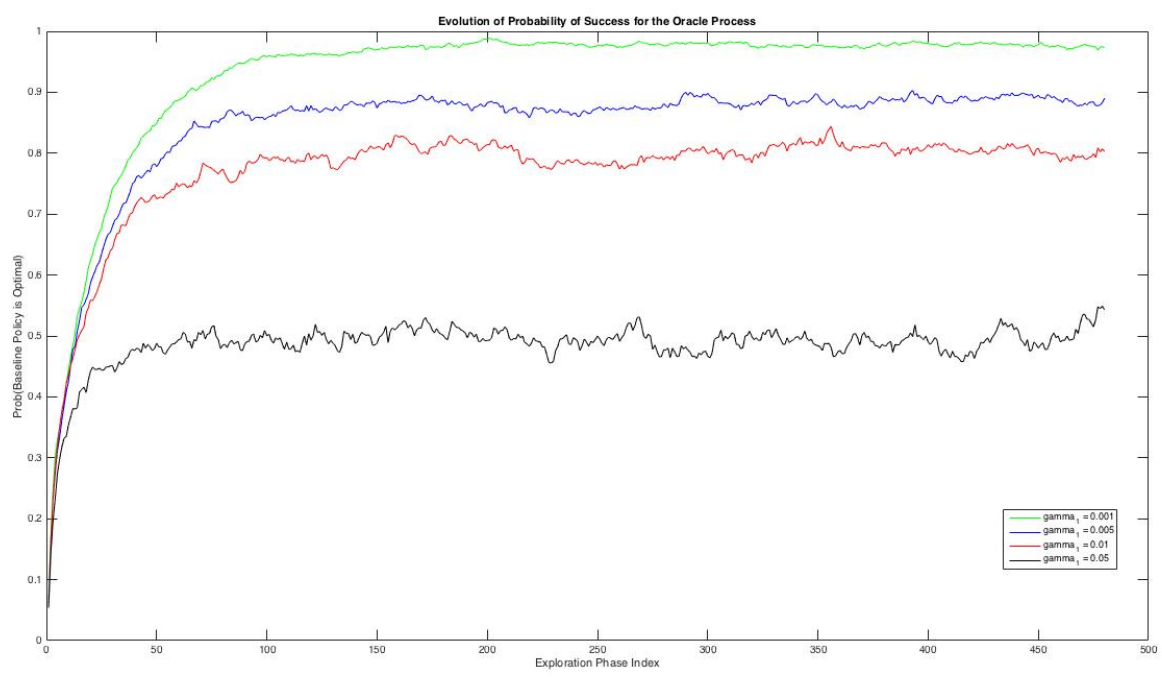

\begin{tabular}{|c|c|}
\hline & $P\left(\pi^{480} \in \Pi^{O P T}\right)$ \\
\hline$\gamma_{1}=0.05$ & 0.543 \\
\hline$\gamma_{1}=0.01$ & 0.803 \\
\hline$\gamma_{1}=0.005$ & 0.89 \\
\hline$\gamma_{1}=0.001$ & 0.973 \\
\hline
\end{tabular}




\section{Numerical Results for Algorithm B}

Algorithm B performed well in the toy example. A plot of its numerical results can be seen below. (The plot uses the label Algorithm A, but this was produced using Algorithm B.) 500 trials were conducted for each value of $\gamma_{1}$. Note that at exploration phase 31, the plots associated with algorithm B experience a downward crash, followed by an upward slope. This owes to the fact that in the first 30 exploration phases, the player does not have a cutoff estimate, and so uses a different policy update rule than in periods after the $30^{t h}$.

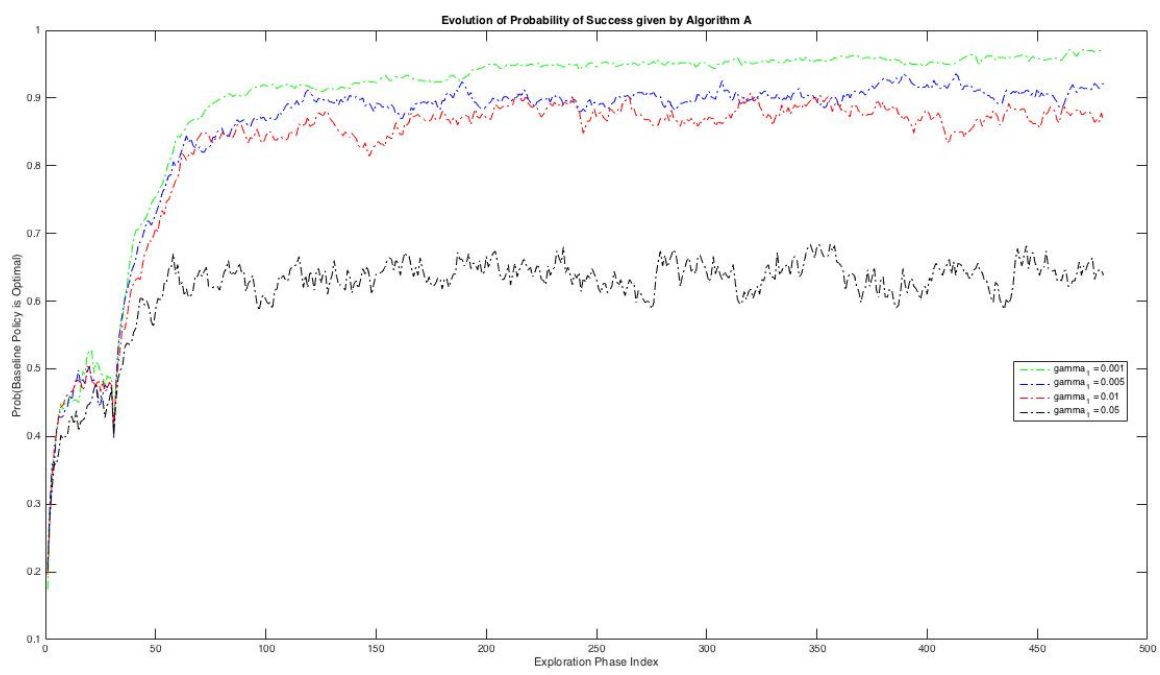

\begin{tabular}{|c|c|}
\hline & $P\left(\pi^{480} \in \Pi^{O P T}\right)$ \\
\hline$\gamma_{1}=0.05$ & 0.638 \\
\hline$\gamma_{1}=0.01$ & 0.866 \\
\hline$\gamma_{1}=0.005$ & 0.922 \\
\hline$\gamma_{1}=0.001$ & 0.972 \\
\hline
\end{tabular}




\section{Discrepancies}

It appears as though the general order is preserved: i.e. lower levels of $\gamma_{1}$ yield higher probabilities of success, especially for greater exploration phase indices. However, the vertical distance between curves appears to greater for higher values of $\gamma_{1}$.

One cannot help but notice the wide gap between the performance of the comparison Markov chain and algorithm B for greater values of $\gamma_{1}$. A plausible explanation for this is that the parameters selected-notably, $\gamma_{1}$ itself-determine whether or not the comparison will be good. Here, it appears that the comparison falls apart for high values of $\gamma_{1}$.

In the graph below, the blue plots corresponding to $\gamma_{1}=0.005$ have been removed, since they overlap partly with the red plots corresponding to $\gamma_{1}=0.01$.

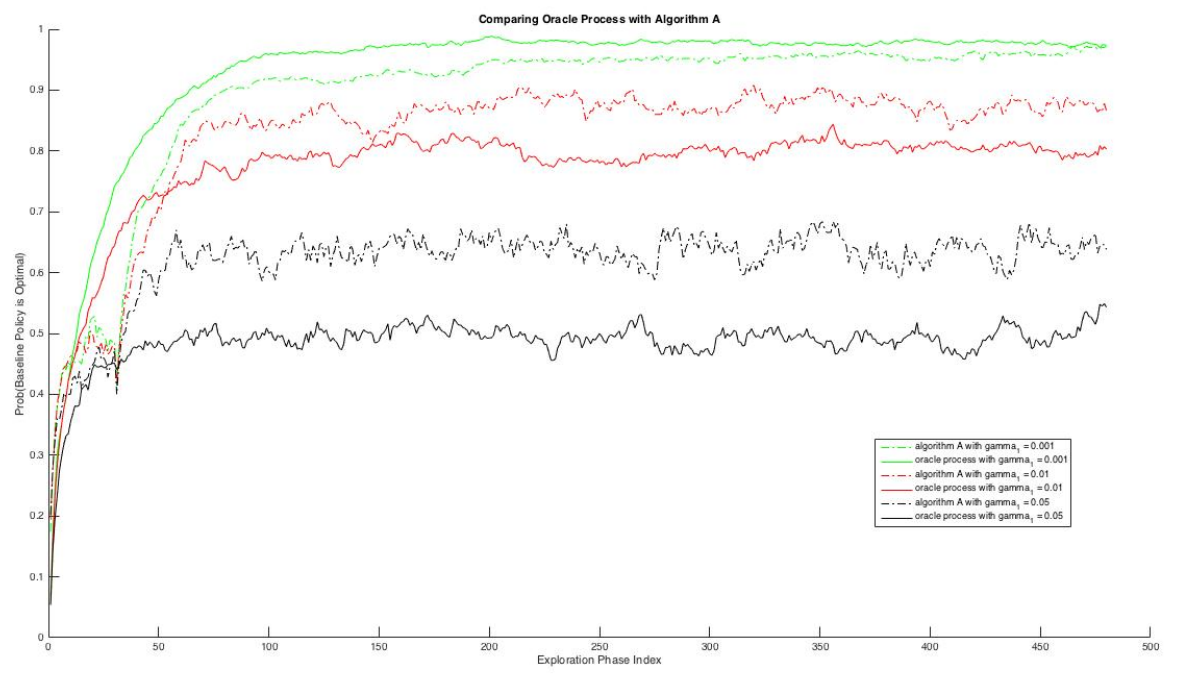




\section{Chapter 5}

\section{Summary and Conclusions}

In this thesis, we presented a decentralized learning algorithm applicable to any finite decentralized controlled Markov problem with an expected discounted cost criterion, and established its convergence to optimal solutions with arbitrarily high probability. In particular, our algorithm improves on Algorithm 1 from [1], which had established convergence to person-by-person-optimality.

During our studies, we also had an intermediate algorithm, where a separator/cutoff parameter $\Lambda$ was provided externally to play the role of $\Lambda_{B}^{i}$ in the Algorithm B (e.g., for setups when an upper bound on an optimal policy and a lower bound on NEXT can be established off-line). For such a case, we established an almost sure convergence result to optimality, similar to such results in simulated annealing and Theorem 1(iii) of [1]. This has not been included in the thesis.

As future work, we wish to generalize our results to a setup where the updates by the decision makers are not synchronous; that is, when controllers have individual, random exploration phase lengths rather than common, deterministic ones. 


\section{Bibliography}

[1] G. Arslan and S. Yüksel. Decentralized Q-learning for stochastic teams and games. IEEE Transactions on Automatic Control, 62(4):1545-1558, 2017.

[2] V. Borkar. Reinforcement learning in Markovian evolutionary games. Advances in Complex Systems, 5(1):55-72, 2002.

[3] C. Claus and C. Boutilier. The dynamics of reinforcement learning in cooperative multiagent systems. In Proceedings of the Tenth Innovative Applications of Artificial Intelligence Conference, Madison, Wisconsin, pages 746-752, July 1998.

[4] V.P. Crawford and H. Haller. Learning how to cooperate: Optimal play in repeated coordination games. Econometrica, 58(3):571-595, 1990.

[5] B. Hajek. Cooling schedules for optimal annealing. Mathematics of Operations Research, 13(2):311-329, 1988.

[6] O. Hernandez-Lerma and J. B. Lasserre. Discrete-Time Markov Control Processes: Basic Optimality Criteria. Springer, 1996.

[7] D. S. Leslie and E. J. Collins. Convergent multiple-timescales reinforcement learning algorithms in normal form games. Ann. Appl. Probab., 13(4):1231-1251, 
November 2003.

[8] J. R. Marden, H. P. Young, G. Arslan, and J. S. Shamma. Payoff-based dynamics for multiplayer weakly acyclic games. SIAM Journal on Control and Optimization, 48(1):373-396, 2009.

[9] S. Sen, M. Sekaran, and J. Hale. Learning to coordinate without sharing information. In Proceedings of the 12th National Conference on Artificial Intelligence, pages 426-431, 1994.

[10] Y. Shoham, R. Powers, and T. Grenager. If multi-agent learning is the answer, what is the question? Artificial Intelligence, 171(7):365-377, 2007.

[11] R. S. Sutton and A. G. Barto. Reinforcement Learning: An Introduction. MIT Press, MA, 1998.

[12] J.N. Tsitsiklis. Asynchronous stochastic approximation and Q-learning. Machine Learning, 16(3):185-202, 1994.

[13] X. Wang and T. Sandholm. Reinforcement learning to play an optimal nash equilibrium in team Markov games. In Proceedings of the 15th International Conference on Neural Information Processing Systems, NIPS'02, pages 16031610, 2002.

[14] C. Watkins. Learning from Delayed Rewards. PhD thesis, Cambridge University, 1989.

[15] C. Watkins and P. Dayan. Q-Learning. Machine Learning, 8(3):279-292, 1992.

[16] H. P. Young. The evolution of conventions. Econometrica, 61(1):57-84, 1993. 
[17] S. Yüksel. Control of Stochastic Systems. [Accessed August 24, 2018]. Available Online:

http://www.mast. queensu.ca/ math472/Math472872LectureNotes.pdf. Lecture Notes.

[18] S. Yüksel and T. Başar. Stochastic Networked Control Systems. Birkhäuser, 2013. 